\title{
Atomic Parity Nonconservation and Nuclear Anapole Moments
}

\author{
W. C. Haxton \\ Institute for Nuclear Theory, Box 351550, and Department of Physics, \\ University of Washington, Seattle, WA 98195, and \\ Department of Physics, University of California, Berkeley, CA 94720 \\ C. E. Wieman \\ JILA and \\ Department of Physics, University of Colorado, Boulder, CO 80309-0440
}

KEYWORDS: anapole moment, atomic parity nonconservation, radiative corrections, weak interactions

\begin{abstract}
Anapole moments are parity-odd, time-reversal-even moments of the E1 projection of the electromagnetic current. Although it was recognized, soon after the discovery of parity violation in the weak interaction, that elementary particles and composite systems like nuclei must have anapole moments, it proved difficult to isolate this weak radiative correction. The first successful measurement, an extraction of the nuclear anapole moment of ${ }^{133} \mathrm{Cs}$ from the hyperfine dependence of the atomic parity violation, was obtained only recently. An important anapole moment bound in $\mathrm{Tl}$ also exists. We discuss these measurements and their significance as tests of the hadronic weak interaction, focusing on the mechanisms that operate within the nucleus to generate the anapole moment. The atomic results place new constraints on weak meson-nucleon couplings, ones we compare to existing bounds from a variety of $\vec{p}-p$ and nuclear tests of parity nonconservation.
\end{abstract}

\section{CONTENTS}

INTRODUCTION . . . . . . . . . . . . . . . . . . . . . . . 2

ANAPOLE MOMENTS AND ATOMS . . . . . . . . . . . . . . . . . . . . . . . . . . . . . . . . 5

Electromagnetic Moments . . . . . . . . . . . . . . . . . . . . . . 5

Atoms and the Generalized Siegert's Theorem . . . . . . . . . . . . . . . . . . . 6

The Spin-Dependent Atomic Interaction . . . . . . . . . . . . . . . . . . . . . . . . . . 8

EXPERIMENTS ............................. . . 10

The Cs Experiment: Techniques . . . . . . . . . . . . . . . . . . . . . . 10 
Annu. Rev. Nuclear and Particle Science 20011 1056-8700/97/0610-00

The Cs Experiment: The Apparatus . . . . . . . . . . . . . . . . . . . . 14

The Cs Experiment: Data and Results . . . . . . . . . . . . . . . . . . . . . . 15

The $T l$ Experiment . . . . . . . . . . . . . . . . . . . . . . . 17

ANAPOLE MOMENTS AND HADRONIC PNC . . . . . . . . . . . . . . . . . . . . 19

Extracting the Anapole Moment . . . . . . . . . . . . . . . . . . . . . . . 20

The Hadronic Weak Interaction . . . . . . . . . . . . . . . . . . . . . . . . . . . . . 21

Weak Meson-Nucleon Couplings and the Anapole Moment . . . . . . . . . . . . . . . . . 24

WEAK COUPLING CONSTRAINTS . . . . . . . . . . . . . . . . . . . . . . . 29

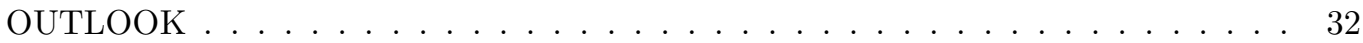

\section{INTRODUCTION}

Until 1957 physicists assumed that the fundamental laws of nature did not distinguish between left and right. However in that year, following a suggestion by Lee and Yang (11), experimenters discovered that the weak force governing processes such as muon decay and nuclear beta decay violated mirror symmetry maximally (to the accuracy of the measurements) (2). Very soon afterwards Vaks and Zeldovich (33) noted that weak interactions would then modify the electromagnetic couplings to elementary particles (as well as composite systems), allowing them to have a new, parity-violating moments, called anapole moments, in addition to their familiar parity-conserving ones (e.g., the charge and magnetic moments).

This new moment has a number of curious properties. It vanishes when probed by real photons, and thus must be tested in processes where a virtual photon is exchanged. Thus, for example, the anapole moment of a nucleus can be probed in electron scattering and can influence the energies of bound electrons in an atom, but cannot be measured through direct interactions with an electric field (unlike magnetic moment interactions in a static $\vec{B}$ field). The resulting electron-nucleus interaction in an atom is pointlike, thus mimicking the short-range tree-level weak interaction induced by $Z^{0}$ exchange between atomic electrons and the nucleus. The atomic cloud feels the nuclear anapole moment only to the extent that the orbiting electrons penetrate the nucleus. The anapole moment is an electric dipole coupling that is nuclear spin-dependent: it is this spin dependence, as we will see, that allows anapole effects to be separated from tree-level weak interactions. Finally, the anapole moment is in general one of a larger class of weak radiative corrections. The anapole interaction - one diagram is given in Fig. 1a - is thus accompanied by other radiative corrections that do not correspond to virtual photon exchange, such as Fig. 1b. It is the sum of all such diagrams that contribute to physical observables. It follows that the anapole moment is not a measurable, that is, not separately a gauge-invariant quantity. (However, we will later see that the dominate contribution to nuclear anapole moments is well defined and separately gauge invariant.) 
a)

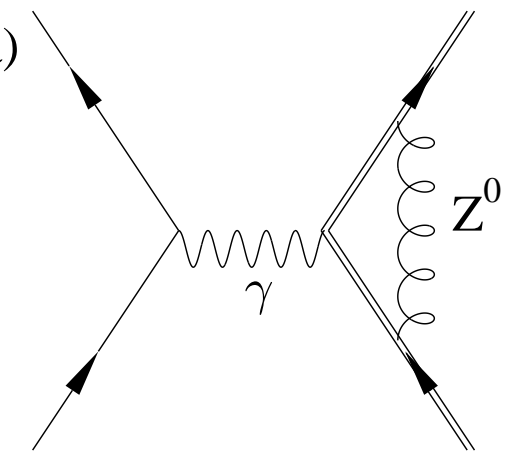

b)

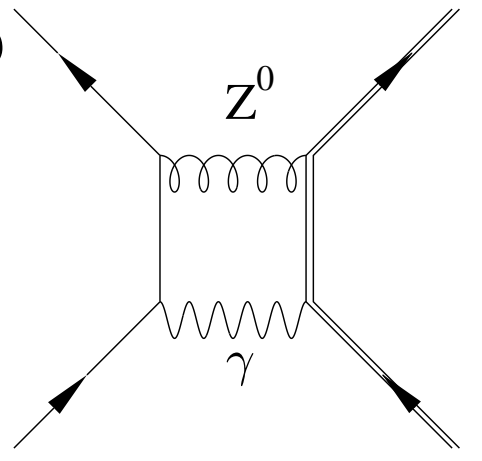

Figure 1: Weak radiative corrections to electron-proton scattering include anapole contributions (a) as well as conributions that do not correspond to a single virtual photon exchange (b).

Our primary focus in this review is the nuclear anapole moment contribution to atomic parity nonconservation (PNC). Exquisitely precise (sub 1\%) measurements of atomic PNC have become possible in the past few years (伍). The primary focus of these studies has been to obtain precise values of the strength of direct $Z^{0}$ exchange between electrons and the nucleus. The PNC effects are dominated by the exchange involving an axial $Z^{0}$ coupling to the electrons and a vector coupling to the nucleus. The nuclear coupling is thus coherent, proportional to the nuclear weak vector charge (approximately the neutron number), and independent of the nuclear spin direction. It is widely recognized that these atomic measurements are important tests of the standard electroweak model and its possible extensions, complementing what has been learned at high energy accelerators that directly probe physics near the $Z^{0}$ pole $(5,6,6)$. The comparison between precision measurements at atomic energies and accelerator energies could reveal the subtle influence of new interactions beyond the standard model.

At low energies one expects weak radiative corrections, including the anapole contribution, to interfere with the dominant tree-level weak amplitude, producing corrections to observables of relative size $\alpha \sim 1 \%$, where $\alpha$ is the fine structure constant. Therefore identifying the anapole contribution in weak processes would seem a daunting task. The possibility that nuclear spin-dependent PNC measurements in heavy atoms might prove an exception was first pointed out two decades ago by Flambaum, Khriplovich, and collaborators (8). Such spin-dependent PNC effects involve a vector coupling to the electrons and an axial coupling to the nucleus. In this case the contribution of $Z^{0}$ exchange is considerably reduced because the nuclear interaction is no longer coherent, but instead samples, in a 
naive picture, only the spin of the last, unpaired nucleon within an odd- $A$ nucleus. Additional suppression comes from the small $Z^{0}$ vector coupling to the electron, $\sim\left(4 \sin ^{2} \theta_{W}-1\right) / 2 \sim-0.05$. In contrast, the anapole moment is enhanced in heavy nuclei, growing as $A^{2 / 3}$, where $A$ is the atomic number, and thus increasing in proportion to the nuclear surface area. The net result is the surprising conclusion that the anapole contribution - the weak radiative "correction" - will in fact dominate over the tree-level direct $Z^{0}$ spin-dependent contribution for nuclei heavier than $A \sim 20$.

The principal motivation for this review was the recent successful determination of the nuclear spin-dependent contribution to atomic PNC in ${ }^{133} \mathrm{Cs}$ (9). The extraction of this contribution from the much larger coherent PNC signal was accomplished by studying the dependence of the PNC signal on the choice of hyperfine level. As the hyperfine differences are quite small, this extraction requires detailed control of possible sources of systematic error. Over a decade ago the Colorado group (10) succeeded in measuring PNC effects in ${ }^{133} \mathrm{Cs}$ to $2.2 \%$, providing a tentative identification of the anapole moment. Six years ago the Seattle (11) and Oxford (12) groups achieved an accuracy of $1.2 \%$ and $2.9 \%$, respectively, in PNC measurements in natural Tl, resulting in bounds on the anapole moment in this nucleus. Finally, in 1997 a definite measurement was made: the Colorado group of Wood et al. (9) reported PNC measurements in ${ }^{133} \mathrm{Cs}$ at the $0.35 \%$ level, from which a $7 \sigma$ nuclear spin-dependent PNC signal was extracted. This spin dependence is clearly well above the expected signal from $Z^{0}$ exchange alone but compatible with the enhancement expected from anapole effects.

As we will describe in this review, a nucleus generates an anapole moment through the weak NN interaction, which operates at long range in the nucleus by meson exchange, where one meson-nucleon vertex is strong and the other weak (13, 14). Such interactions admix into the nuclear ground state odd-parity amplitudes and also induce new PNC nuclear currents. Thus the exquisite precision now achieved in atomic PNC studies has opened up a new window on the hadronic weak interaction. This interaction has proven more elusive than the weak interactions involving leptons. Whereas the charge-changing hadronic weak interactions can be studied in strangeness- or charm-changing decays, the standard model predicts that neutral-current interactions do not change flavor. Thus hadronic interactions mediated by the $Z^{0}$ can only be studied in NN interactions, where PNC must be exploited to separate this contribution from much larger strong and electromagnetic effects. This is a difficult task, and only a few hadronic experiments have achieved the requisite precision and even fewer (notably those done with $\vec{p}+p$ scattering or in certain light nuclei with special properties) can be interpreted, reasonably free of nuclear structure uncertainties. The importance of atomic anapole moment measurements is not only that they supplement the hadronic data, but also that they are sensitive to long-range pion-exchange PNC interactions where the effects of the neutral current can be isolated. We will see, however, that the extraction of weak coupling constant constraints from anapole moments are requires 
some nonrivial nuclear physics analysis.

The plan of this review is as follows. In Section 2 we discuss general properties of anapole moments, the associated current distribution, and the spin-dependent interaction nuclear anapole moments generate in atoms. In Sections 3 and 4 we summarize the experimental status of atomic anapole moment measurements and the progress theory has made in estimating the size of nuclear anapole moments from an underlying model of the hadronic weak interaction. In Section 5 we compare weak meson-nucleon coupling constraints obtained from the ${ }^{133} \mathrm{Cs}$ anapole result and $\mathrm{Tl}$ limits with similar constraints obtained from PNC nuclear observables such as $\vec{p}+p$ scattering and the circular polarization of gamma rays emitted from ${ }^{18} \mathrm{~F}$. It becomes clear that, while all measurements are consistent with the broad "reasonable ranges" for PNC meson-nucleon couplings that theorists have defined (13), there is some level of disagreement between various experiments when a global fit is performed. We conclude by discussing prospects for improving our experimental and theoretical knowledge of anapole moments.

\section{ANAPOLE MOMENTS AND ATOMS}

In this section we discuss general properties of nuclear anapole moments and the interactions they induce in atoms like ${ }^{133} \mathrm{Cs}$ and $\mathrm{Tl}$.

\subsection{Electromagnetic Moments}

A standard multipole decomposition (15) of the electromagnetic current groups interactions according to their multipolarity and symmetry properties into charge $\left(\hat{C}_{J}\right)$, transverse electric $\left(\hat{T}_{J}^{e l}\right)$, and transverse magnetic $\left(\hat{T}_{J}^{\text {mag }}\right)$ operators, where $J$ denotes the multipole rank. Static moments correspond to diagonal matrix elements of these multipole operators. From the transformation properties of the ordinary electromagnetic current operator under parity $(P)$ and time-reversal $(T)$

$$
\begin{array}{r}
\hat{P} J^{\mu}(\vec{x}, t) \hat{P}^{-1}=J_{\mu}(\vec{x}, t) \\
\hat{T} J^{\mu}(\vec{x}, t) \hat{T}^{-1}=J_{\mu}(\vec{x},-t),
\end{array}
$$

and the constraint of hermiticity, it is readily verified that the non-zero moments arise from matrix elements of the even- $J$ projections of $C_{J}$ and the odd- $J$ projections of $T_{J}^{\text {mag }}(15)$.

More possibilities arise if one turns on the weak interaction, which introduces both parity- and $\mathrm{CP} / \mathrm{T}$-violating terms. Thus it is interesting to classify possible static moments more generally, without the assumption that the underlying Hamiltonian conserves electromagnetic symmetries. The results in Table 1 show that, in addition to the ordinary monopole charge and magnetic dipole moments, two new dipole moments then arise. One of these, corresponding to the expectation value of the $C_{1}$ multipole, requires both parity and $C P / T$ violation. This is the electric dipole moment which, while allowed in the standard model due to $C P$ violation in 
Table 1: Transformation properties of electromagnetic moments

\begin{tabular}{cccc}
\hline \hline$J$ & $C_{J}$ & $T_{J}^{e l}$ & $T_{J}^{\text {mag }}$ \\
\hline 0 & $P$-even, $T$-even & & \\
1 & $P$-odd, $T$-odd & $P$-odd, $T$-even & $P$-even, $T$-even \\
2 & $P$-even, $T$-even & $P$-even, $T$-odd & $P$-odd, $T$-odd \\
3 & $P$-odd, $T$-odd & $P$-odd, $T$-even & $P$-even, $T$-odd \\
\hline
\end{tabular}

the quark mass matrix and in the $\theta$ term, has yet to be detected experimentally. The second, an E1 moment, requires only parity violation. This is the anapole moment.

These monopole and dipole moments must therefore arise in the most general expression for the matrix element of a conserved four-current for a spin- $\frac{1}{2}$ particle

$$
\begin{aligned}
\bar{U}\left(p^{\prime}\right) J^{\mu}(q) U(p)= & \bar{U}\left(p^{\prime}\right)\left(F_{1}\left(q^{2}\right) \gamma^{\mu}-i \frac{F_{2}\left(q^{2}\right)}{2 M} \sigma^{\mu \nu} q_{\nu}\right. \\
& \left.+\frac{a\left(q^{2}\right)}{M^{2}}\left(\not q q^{\mu}-q^{2} \gamma^{\mu}\right) \gamma_{5}-i \frac{d\left(q^{2}\right)}{M} \sigma^{\mu \nu} q_{\nu} \gamma_{5}\right) U(p) .
\end{aligned}
$$

The two vector terms define the charge $F_{1}\left(q^{2}\right)$ and magnetic $F_{2}\left(q^{2}\right)$ form factors. The axial terms that follow are the anapole and electric dipole terms, respectively. The anapole term reduces in the nonrelativistic limit to

$$
\begin{aligned}
\frac{a\left(q^{2}\right)}{M^{2}}\left(\not q q^{\mu}-q^{2} \gamma^{\mu}\right) \gamma_{5} & \rightarrow \frac{a\left(q^{2}\right)}{M^{2}} \vec{q}^{2}(\vec{\sigma}-\hat{q} \hat{q} \cdot \vec{\sigma}) \\
& =\frac{a\left(q^{2}\right)}{M^{2}} \vec{q}^{2} \vec{\sigma}_{\perp},
\end{aligned}
$$

showing that the current is transverse and spin dependent. We define the anapole operator as

$$
\hat{A}_{1 \lambda}=a(0) \sigma_{1 \lambda} .
$$

Weak radiative corrections that modify physical processes involving elementary fermions thus include anapole moment contributions. One interesting example is the Majorana neutrino, where the identity under particle-antiparticle conjugation requires the magnetic and electric dipole moments to vanish, but permits an anapole moment (16). Yet the interest in anapole moments would likely have remained largely theoretical were it not for the realization that they might play a significant role in atomic PNC expriments.

\subsection{Atoms and the Generalized Siegert's Theorem}

A generalization of the anapole operator is helpful for the case of a composite system like a nucleus, where the current operator and wave functions are modified 
by the interactions among the constituents, inducing parity admixtures in the ground state and multi-body currents. In the standard multipole decomposition the $E 1$ projection of the current is expressed in terms of the multipole operator $\hat{T}_{1 \lambda}^{e l}$

$$
\vec{J}_{1 \lambda}(\vec{q}) \stackrel{E 1}{\rightarrow}-i \sqrt{6 \pi} \hat{T}_{1 \lambda}^{e l}(q)
$$

where

$$
\hat{T}_{1 \lambda}^{e l}(q)=\frac{1}{q} \int d^{3} x \vec{J}(\vec{x}) \cdot \vec{\nabla} \times\left(j_{1}(q x) \vec{Y}_{11 \lambda}\left(\Omega_{x}\right)\right) .
$$

It is well known that current conservation places constraints on the matrix elements of $\hat{T}_{J \lambda}^{e l}(q)$. A familiar example is the long-wavelength limit of $\hat{T}_{1 \lambda}^{e l}(q)$ generated from the ordinary, parity-conserving electromagnetic current. The operator then is $\vec{p} / M$, which is of order $v / c$, where $v$ is the nucleon velocity. It can be shown that the exchange-current contributions to the vector three-current are also of order $v / c$. For realistic models of the nucleus which account for the interactions among the nucleons, in general we lack a prescription for constructing interactions and currents consistently - and for renormalizing them appropriately to take into account the limited Hilbert spaces employed in nuclear models. As a result, there will be errors in evaluations of $\hat{T}_{1 \lambda}^{e l}$, owing to the imperfect construction of the current, that are necessarily of leading order in the velocity, $v / c$.

Siegert (17) showed that the situation could be greatly improved by exploiting the continuity equation

$$
\vec{\nabla} \cdot \vec{J}(\vec{x})=-i[H, \rho(\vec{x})]
$$

to rewrite $\hat{T}_{J}^{e l}$, in the long wavelength limit, entirely in terms of the charge operator. This generates the familiar dipole form of $\hat{T}_{J}^{e l}$, proportional to $\omega \vec{r}$, where $\omega$ is the energy transfer. The importance of this rewriting is that the charge operator, which is of order $(v / c)^{0}$, has exchange current corrections only of order $(v / c)^{2}$, or of relative size $\sim 1 \%$. Thus the Siegert's form of $\hat{T}_{J}^{e l}$, in which the constraints of current conservation are fully exploited, is a far more controlled operator for use in nuclear calculations.

The analogous situation arises for the anapole moment. While there are a variety of forms of the anapole operator that are equivalent up to terms that vanish by current conservation, these forms are not equivalent operationally in realistic calculations because of model violations of current conservation. The simple, long-wavelength form of Siegert's theorm doesn't address the question of moments because it generates an operator proportional to $\omega$, which then vanishes for a diagonal matrix element. Fortunately the generalization of Siegert's theorem (18) exists: at arbitrary $q$ one can write $\hat{T}_{J}^{e l}(q)=\hat{S}_{J}(q)+\hat{R}_{J}(q)$, where all components of the electromagnetic current that are constrained by current conservation have been isolated in $\hat{S}_{J}$ and expressed as a commutator of the charge operator with the nuclear Hamiltonian. All such terms then vanish for a static moment. The resulting generalized Siegert's form of $\hat{T}_{J}^{e l}$ appropriate for diagonal matrix elements 
is

$$
\left.\hat{T}_{1 \lambda}^{e l}(q)\right|_{\text {diagonal }} \stackrel{\vec{q} \rightarrow 0}{\rightarrow}-\frac{i \vec{q}^{2}}{9 \sqrt{6 \pi}} \int d^{3} r^{2}\left[J_{1 \lambda}(\vec{r})+\sqrt{2 \pi}\left(Y_{2}\left(\Omega_{r}\right) \otimes J_{1}(\vec{r})\right)_{1 \lambda}\right] .
$$

From Eqs. (5) and (8) we have the appropriate threshold form of the current operator. The anapole operator is defined relative to the current operator as in Eqs. (3) and (4), yielding immediately (19)

$$
\hat{A}_{1 \lambda}=-\frac{M^{2}}{9} \int d^{3} r r^{2}\left[J_{1 \lambda}(\vec{r})+\sqrt{2 \pi}\left(Y_{2}\left(\Omega_{r}\right) \otimes J_{1}(\vec{r})\right)_{1 \lambda}\right]
$$

Other forms of the anapole operator are more commonly used, for example (20)

$$
\hat{A}_{1 \lambda}=-\pi \int d^{3} r r^{2} J_{1 \lambda}(\vec{r}) .
$$

Apart from the normalization, which is a matter of convention, this form will be equivalent to Eq. (9) only if the nuclear model is sufficiently simple that exact current operators can be constructed. This would be the case for a simple central potential model, such as an independent-particle harmonic oscillator, for which the appropriate current operator is that of a free particle. This would also be the case for an independent-particle model with spin-orbit and orbital potentials of the form $\vec{\sigma} \cdot \vec{\ell}$ or $\vec{\ell} \cdot \vec{\ell}$, provided that the additional contributions to the current obtained by minimal substitution, $\vec{p} \rightarrow \vec{p}-e \vec{A}$ into $\vec{\ell}=\vec{r} \times \vec{p}$, are included in the calculation (21). But in other, more realistic treatments of the nuclear physics, Eq. (9) is the unique form that fully enforces the constraints of current conservation, regardless of the complexity of the current operators.

Figure 2 gives a classical picture of the anapole moment as a current winding within the nucleus. Although the currents on the inner and outer sides of the torus oppose one another, there is a net contribution to Eq.(9) because of $r^{2}$ weighting of the current, leading to an anapole moment that points upward. (Note the sign in Eq. (9).) The current distribution drawn in Fig. 2 is odd under reversal of parity, as is the ordinary $J_{1 \lambda}^{e m}$. Thus it is easy to see that the corresponding anapole moment is a parity-odd operator. If, however, the current has a chirality - a small "pitch" corresponding to a left- or right-handed winding that would signal PNC a parity-even contribution to the operator would be induced. This is the analog of evaluating Eq. (9) with the axial-vector current induced by the weak interaction, leading to an operator component that would have a nonzero expectation value for a parity-conserving ground state. Similarly, the anapole moment associated with $J_{1 \lambda}^{e m}$

would have a nonzero expectation value if the nuclear ground state contains parityodd components induced by the weak PNC component of the $N N$ interaction.

\subsection{The Spin-Dependent Atomic Interaction}

The potential felt by atomic electrons due to the nuclear anapole moment is generated in the standard way by the dipole current - dipole current interaction. As 


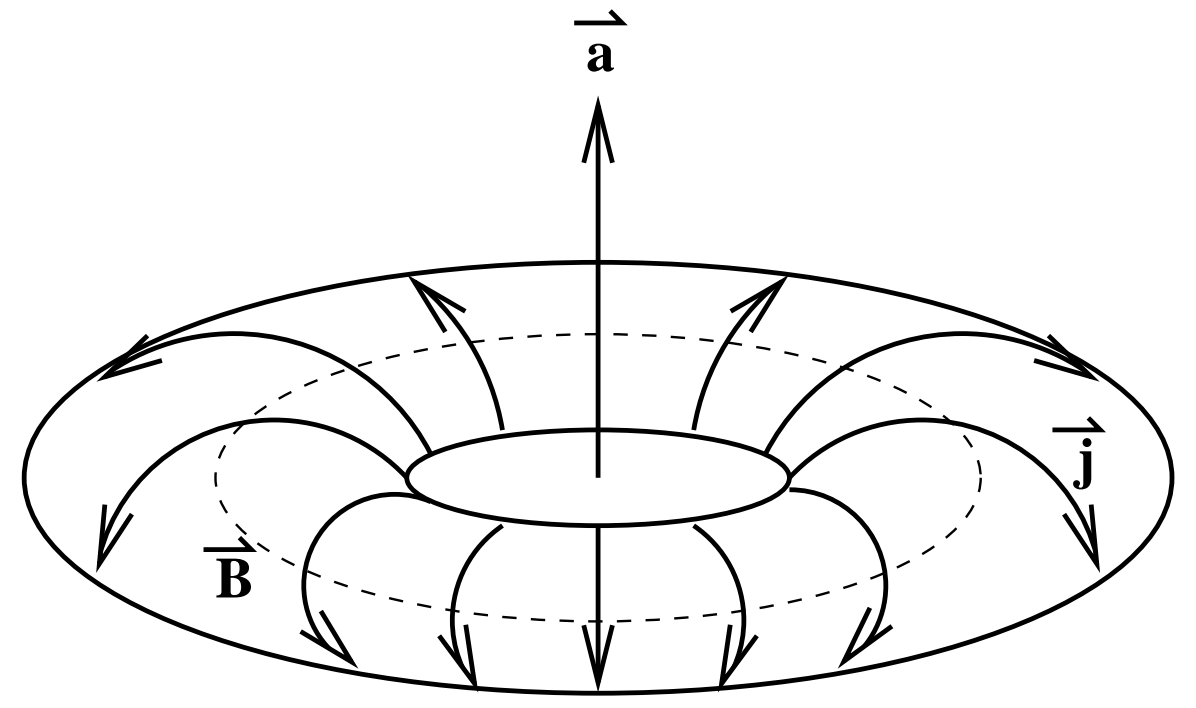

Figure 2: A toroidal current winding that would correspond to a nonzero anapole operator in Eq. (9).

the $\vec{q}^{2}$ appearing in the nuclear current cancels the photon propagator for a static interaction, this weak interaction has the contact form

$$
H_{W}^{s . d .}=\frac{G_{F}}{\sqrt{2}} \kappa \vec{\alpha} \cdot \vec{I} \rho(\vec{r}),
$$

where $\vec{I}$ and $\rho(\vec{r})$ are the nuclear spin and density, and where $\vec{\alpha}$ is the Dirac matrix acting on the electrons. The superscript s.d. denotes that this is the nuclear spin-dependent contribution to the atomic PNC interaction. The portion of this interaction generated by the nuclear anapole moment is then

$$
\kappa_{\text {anapole }}=\frac{4 \pi \alpha \sqrt{2}}{M^{2} G_{F}} \frac{<I\left\|\hat{A}_{1}\right\| I>/ e}{<I|| \hat{I} \| I>}
$$

where $\|$ denotes a matrix element reduced in angular momentum and $\alpha$ is the fine structure constant. The reduced matrix element of $\hat{I}$ is $\sqrt{I(I+1)(2 I+1)}$. [Note that elsewhere in the literature another definition of $\kappa$ is more commonly used, one that associates the nuclear ground state with a single-particle level of orbital angular momentum $\ell$ and spin $I$. For example, the $\kappa$ used in Ref. (8) is obtained by dividing ours by the factor $(-1)^{I+1 / 2+\ell}(I+1 / 2) /(I(I+1))$.]

We have already noted that $\kappa_{\text {anapole }}$ is just one of the contributions to $\kappa$ : isolating this anapole contribution would be clearly easier if $\kappa_{\text {anapole }}$ were the dominant such contribution. It was the important observation of Flambaum and Khriplovich (8) that the $A^{2 / 3}$ growth of nuclear anapole moments, already implicit in the $r^{2}$ weighting of the current in Eq. (9), would lead to such dominance in heavy atoms. It is 
also clear that, if $\kappa_{\text {anapole }}$ could be extracted from atomic measurements, relating this quantity to the underlying sources of hadronic PNC involves specifying the PNC currents that contribute to Eq. (9), as well as the PNC admixtures in the nuclear ground state wave function through which the ordinary electromagnetic current operator will generate nonvanishing matrix elements of $\hat{A}_{1 \lambda}$. We will defer this question to Section 4, focusing now on the experimental progress that has produced values and limits for $\kappa$.

\section{EXPERIMENTS}

The idea of the anapole moment languished for many years after the early work of Zeldovich and Vaks. In 1973 Henley, Huffman, and Yu (22) - who were unaware of the Zeldovich paper - noted that the anapole moment would contribute to PNC observables in high energy electron scattering off the proton. Then a revival of interest in the anapole moment occurred in 1980 when its possible relevance to ongoing experiments on atomic PNC was noticed (8). In these experiments the tiny parity-violating mixing of $S$ and $P$ states induced by the coherent, nuclearspin-independent $Z^{0}$ coupling to the nucleus was being measured (23). As both the coherence and the increased electronic overlap with the nucleus lead to larger PNC effects in heavy atoms, the experiments focused on atoms with $A \gtrsim 100$. The $A^{2 / 3}$ enhancement of the anapole moment makes the spin-dependent contribution to atomic PNC dominant over other vector(electron)-axial(nucleus) sources of PNC in such heavy atoms. Spin-dependent effects of the expected size - a few percent of the spin-independent signal - could, in principle, be extracted from the experiments by precisely comparing the amount of mixing for two different hyperfine components, i.e., electronic transitions that differ only in the orientation of the nuclear spin.

Nevertheless, the spin-dependent PNC effects are painfully small, corresponding to state mixings on the order of parts in $10^{13}$. That level of precision was eventually reached in a cesium PNC experiment and nearly reached in experiments with thallium. The isolation of a definite spin-dependent contribution in cesium provided the first confirmation that anapole moments exist.

\subsection{The Cs Experiment: Techniques}

The experiment in cesium is described in detail in Refs. (24). The central idea is to exploit the highly forbidden $6 S$-to- $7 S$ transition in atomic cesium. The strength of the transition depends on the handedness within the excitation region. That handedness is defined by various applied electric, magnetic, and laser fields and reverses with appropriate reversals of those applied fields. This reversal forces any $\mathrm{PNC}$ component to the excitation rate to change sign, thereby altering the excitation rate for the transition.

As the fractional change in the excitation rate is very small, a great deal of work must be done to achieve a signal-to-noise ratio sufficient to see any effect. 


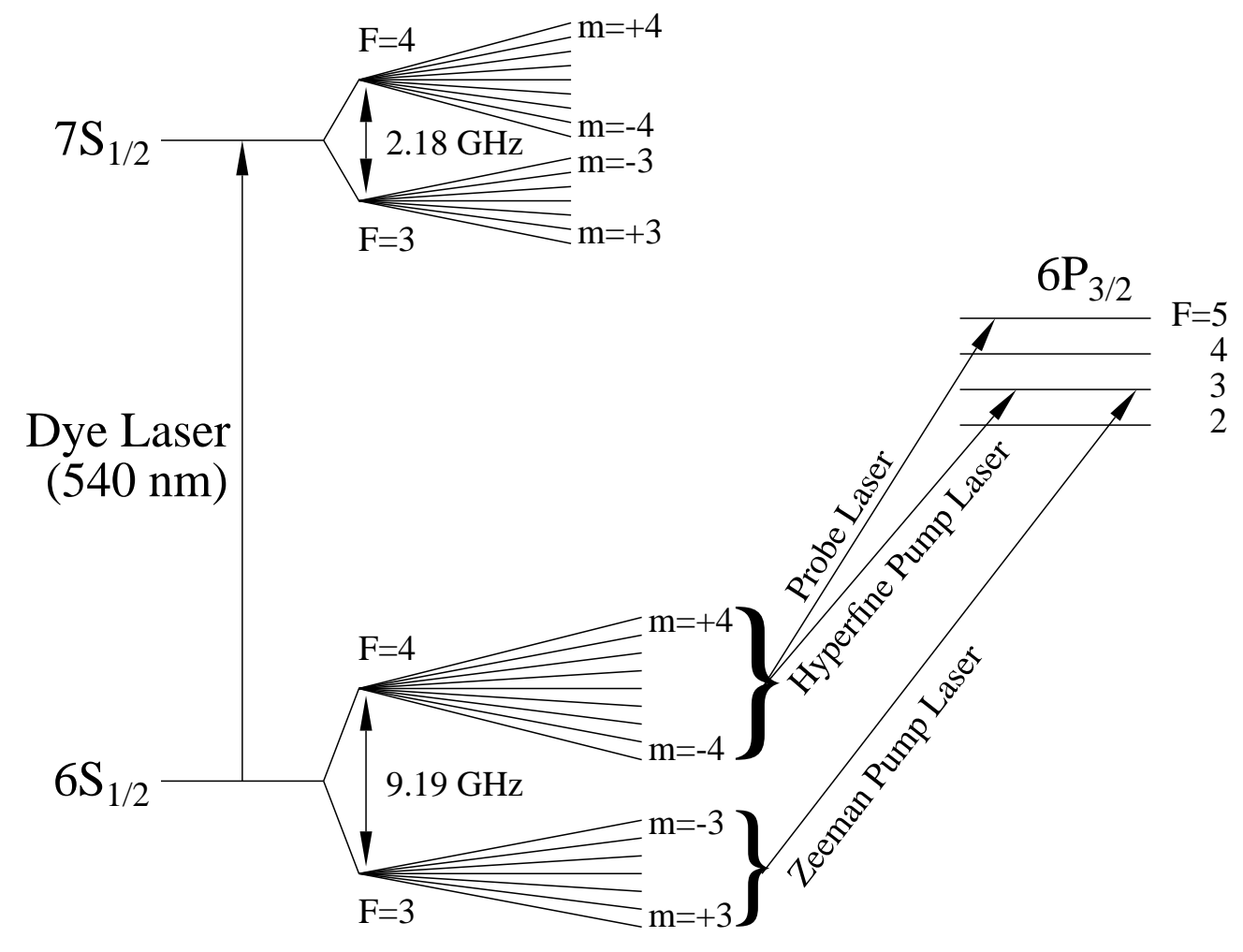

Figure 3: Partial cesium energy-level diagram including the splitting of the $S$ states by the magnetic field. The case of 540-nm light exciting the $\left(F=3, m_{F}=3\right)$ level is shown. Diode lasers 1 and 2 optically pump all of the atoms into the $(3,3)$ level, and laser 3 drives the $6 S_{F=4}\left(F_{\text {det }}\right)$ to $6 P_{F=5}$ transition to detect the $7 S$ excitation. PNC is also measured for excitation from the $(3,-3),(4,4)$, and $(4,-4) 6 S$ levels. The diode lasers excite different transitions for the latter two cases.

Then even more work must be invested to verify that the detected effect is truly a violation of parity and not a spurious signal arising from systematic errors such as imperfect reversals or alignments of the fields that define the handedness of the experiment.

In the absence of electric fields or parity violating interactions, the electric dipole (E1) transition between the $6 S$ and $7 S$ states of the cesium atom (Fig. 3) is forbidden. As the nuclear spin of ${ }^{133} \mathrm{Cs}$ is $I=7 / 2$, these $S_{1 / 2}$ levels combine with the nuclear ground state to form hyperfine states of total angular momentum $F=$ 4 and 3. A PNC interaction mixes a small amount $\left(\sim 10^{-11}\right)$ of the neighboring $6 P_{3 / 2}$ and $7 P_{3 / 2}$ states into the $6 S$ and $7 S$ states: the $P_{3 / 2}$ hyperfine levels have $F=2-5$, so that PNC mixing with both the $F=3$ and 4 hyperfine $S$ states 
takes place. The induced $7 S \leftrightarrow 6 S$ PNC $E 1$ amplitude is thus proportional to the product of the PNC mixing and $E 1$ matrix elements coupling the $S$ and $P$ hyperfine levels (and inversely proportional to the energy differences between the $S$ and $P$ levels). To obtain a measurable observable that is first order in this small amplitude, a d.c. electric field $\vec{E}$ is applied that also mixes $S$ and $P$ states. This field generates an interfering "Stark-induced" $E 1$ transition amplitude $A_{E}$ that is typically $10^{5}$ times larger than the PNC-induced $E 1$ amplitude $A_{P N C}$. A complete analysis of the relevant transition rates between the various hyperfine magnetic states $\left(F, m_{F}\right)$ is given in Ref. (24). These rates involve a straightfoward evaluation of the hyperfine matrix elements for the Stark amplitude, the nuclearspin-dependent PNC Hamiltonian given in Eq. (11), and the spin-independent PNC Hamiltonian for the much stronger coherent interaction with the nucleus,

$$
H_{W}^{s . i .}=\frac{G_{F}}{2 \sqrt{2}} Q_{W} \gamma_{5} \rho(\vec{r})
$$

Here $\gamma_{5}$ is the axial coupling to the electrons, while the tree-level standard-model result for the vector weak charge of a point nucleus is

$$
Q_{W}=Z\left(1-4 \sin ^{2} \theta_{W}\right)-N \sim-N .
$$

We omit the somewhat tedious angular momentum algebra here. To generate a nonzero interference between $A_{P N C}$ and $A_{E}$, which differ by a relative phase of $i$, the $6 S$-to- $7 S$ transition must be excited with an elliptically polarized laser field of the form $\epsilon_{z} \hat{z}+p i \operatorname{Im}\left(\epsilon_{x}\right) \hat{x}$, where the handedness of the polarization $p$ is $\pm 1, \epsilon_{x}$ is the component of the oscillating electric field parallel to the d.c. Stark field $\vec{E}$, and $\epsilon_{z}$ is the oscillating field component in the direction perpendicular to $\vec{E}$. The PNC contribution to the transition rate is measured on both the $6 S_{F=3}$-to- $7 S_{F=4}$ and $6 S_{F=4}$-to- $7 S_{F=3}$ transitions. To a good approximation the only difference between the two transitions is the reversal of the nuclear spin. Thus simply taking the difference between the PNC contributions to the hyperfine transition rates isolates the nuclear-spin-dependent PNC coupling $\kappa$ of Eq. (11).

For both transitions the atoms are initially populated and excited only out of states with extreme values of $m_{F},\left(F=3, m_{F}= \pm 3\right)$ and $\left(F=4, m_{F}= \pm 4\right)$. For these cases and the configuration of electric and magnetic fields shown in Fig. 4, the transition rate is

$$
\begin{aligned}
R & =\left|A_{E}+A_{P N C}\right|^{2} \sim \beta^{2} E_{x}^{2} \epsilon_{x}^{2} C_{1}\left(F, m_{F} ; F^{\prime}, m_{F}^{\prime}\right) \\
& +4 \beta E_{x} \epsilon_{x} p \operatorname{Im}\left(\epsilon_{\mathrm{x}}\right) \operatorname{Im}\left(\mathrm{E} 1_{\mathrm{PNC}}\right) \mathrm{C}_{2}\left(\mathrm{~F}, \mathrm{~m}_{\mathrm{F}} ; \mathrm{F}^{\prime}, \mathrm{m}_{\mathrm{F}}^{\prime}\right)
\end{aligned}
$$

where $\beta$ is the tensor transition polarizability, $E_{x}=|\vec{E}|$ is the d.c. electric field strength, and $C_{1}$ and $C_{2}$ are combinations of Clebsch-Gordan coefficients whose detailed form we have suppressed (24). They depend on the initial- and final-state hyperfine labels $\left(F, m_{F}\right)$ and $\left(F^{\prime}, m_{F}^{\prime}\right)$ and transform as $C_{1}\left(m_{F}\right) \rightarrow C_{1}\left(-m_{F}\right)$ and $C_{2}\left(m_{F}\right) \rightarrow-C_{2}\left(-m_{F}\right)$ under reversal of the magnetic labels. The tiny contribution 
proportional to $A_{P N C}^{2}$ has been neglected as well as the small $6 S-7 S$ parityconserving magnetic dipole transition amplitude $A_{M 1}$. (As discussed later, $A_{M 1}$ can be a source of many systematic errors that must be addressed carefully.)

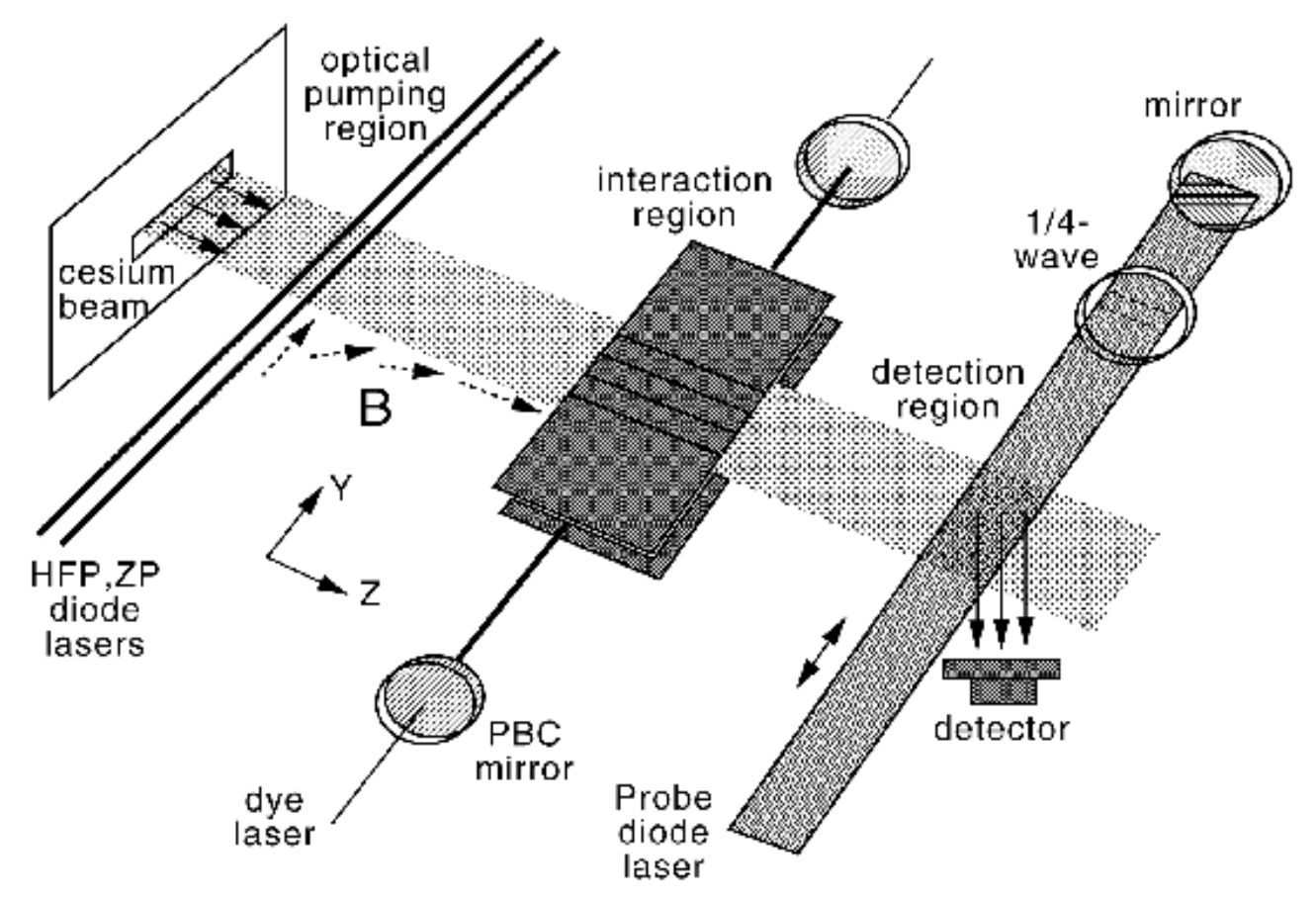

Figure 4: Schematic of the cesium apparatus. In the interaction region $\vec{B}$ is along the $\hat{z}$ axis, $\vec{E}$ is along the $\hat{x}$ axis, and the 540-nm dye laser beam defines the $\hat{y}$ axis.

The experimental quantity of interest is the fractional PNC modulation in the transition rate

$$
\frac{\Delta R}{R}=\frac{2 \operatorname{Im}\left(\epsilon_{\mathrm{x}}\right) \operatorname{Im}\left(\mathrm{E} 1_{\mathrm{PNC}}\right)}{\epsilon_{\mathrm{z}} \beta \mathrm{E}}
$$

that modulates with the reversals of $E, m$, and $p$. In the experiment, there are actually five "parity" reversals because $m_{F}$ is reversed in three different ways. The use of this large number (five) of independent reversals is essential for the detection and elimination of systematic errors, providing a great deal of redundancy for the PNC signal. This redundancy means that although no single reversal is perfect, the product of all the imperfections is far smaller than the PNC signal. Furthermore, the signal modulations accompanying various combinations of field reversals provide additional information about the field reversal imperfections and orientations that help identify potential systematic errors. 


\subsection{The Cs Experiment: The Apparatus}

A simplified schematic of the apparatus used by Wood et al. (9) is shown in Fig. 4. An effusive beam of atomic cesium is produced by a heated oven and collimated with a multichannel capillary array nozzle. The beam is optically pumped into the desired $\left(F, m_{F}\right)$ state by light from diode lasers 1 and 2 (24, 25). The 2$\mathrm{cm}$-wide beam of polarized atoms intersects the 540-nm standing wave (Gaussian diameter, $0.8 \mathrm{~mm}$ ) that is inside a high-finesse $(100,000)$ Fabry-Perot power buildup cavity (PBC). The PBC not only enhances the transition rate, but the standing wave geometry also greatly suppresses the troublesome modulation arising from $A_{E 1}-A_{M 1}$ interference (24). The 540-nm light originates from a dye laser whose frequency is tightly locked to the resonant frequency of the PBC by a high-speed servosystem (26). Before entering the PBC, the dye laser light passes through an intensity stabilizer, an optical isolator, and a polarization control system made up of a half-wave plate, Pockels cell, and adjustable birefringence compensator plate. The ellipticity of the light is controlled by rotating the half-wave plate, and the handedness of the ellipse is reversed by switching the sign of the $1 / 4$ (quarter wavelength) voltage that is applied to the Pockels cell. The intensity stabilizer holds the amount of light transmitted through the cavity constant and hence stabilizes the field inside of the cavity. This field corresponds to about 2.5 $\mathrm{kW}$ of circulating power. The PBC resonant frequency is held at the frequency of the desired atomic transition by a servosystem that translates the input mirror.

The d.c. electric field in the interaction region is produced by applying $\sim 500$ volts between two parallel 5 -cm by $9-\mathrm{cm}$ conducting plates, separated by about $1 \mathrm{~cm}$. The plates are made of flat pieces of Pyrex glass coated with $100 \mathrm{~nm}$ of molybdenum. Both field plates were divided into five electrically separate segments. This division makes it possible to apply small uniform and gradient electric fields along the y-axis for auxiliary diagnostic experiments. The entire buildup cavity and field-plate mounting system is rather elaborate to ensure precise alignment and extreme mechanical stability.

After being excited out of the populated $6 S$ hyperfine level up to the $7 S$ state, an atom will decay by way of the $6 P$ states to the previously empty $6 S$ hyperfine level $\left(F_{\text {det }}\right)$ more than $60 \%$ of the time. The population of $F_{\text {det }}$ is detected 10 $\mathrm{cm}$ downstream of the interaction region using laser florescence. Light from diode laser 3 excites each atom in $F_{\text {det }}$ to the $6 P_{3 / 2}$ state many times. The resulting scattered photons are detected by a silicon photodiode that sits just below the atomic beam. When the $6 S_{F=3}$-to- $7 S_{F=4}$ line is measured, the detection laser drives the $6 S_{F=4}$-to- $6 P_{3 / 2, F=5}$ cycling transition (Fig. 3). About 240 photons per $F_{\text {det }}$ atom are detected. For the $6 S_{F=4}-7 S_{F=3}$ line, the detection transition is the $6 S_{F=3}$-to- $6 P_{3 / 2, F=2}$ cycling transition. This cycle gives about 100 detected photons per $F_{d e t}$ atom. The signal-to-noise ratio for this transition is about $20 \%$ lower. During each half cycle of the most rapid field reversal $(\vec{E})$ and after the switching transient has passed, the detector photocurrent is integrated, digitized, and stored. For each stored value, the computer also records the field and spin 
orientations.

The signal-to-noise ratio needed for this experiment puts extreme requirements on laser stability. A fluctuation in the intensity, frequency, or direction of the light from any of the four lasers will introduce noise in the detected atomic fluorescence. The most extensive control is needed for the dye laser (26), but the requirements on the three diode lasers used for optical pumping and detection are also severe. These requirements motivated substantial development of diode-laser stabilization technology (27). Both optical and electronic feedback were used to lock the frequency of each diode laser to the desired atomic transition using saturated absorption spectrometers.

Extensive and precise control of magnetic fields was required in the experiment. In the optical pumping region, there is a uniform 2.5-G field that must point in the $\hat{y}$ direction (parallel to the pumping laser beams). In the interaction region, a $6.4-G$ field must point precisely along either $\pm \hat{z}$. Between the two regions, the magnetic field must rotate gently enough that the atomic spins follow it adiabatically. Finally, the field must be near zero in the detection region, and it is necessary to precisely reverse the fields in the optical pumping and interaction regions independently without significantly perturbing the fields in the other two regions. The setup required the use of 23 magnetic field coils of various shapes to provide the necessary fields and gradients. Most of these coils were driven with both reversing and nonreversing components of current.

Many additional elements were required to achieve sufficiently precise alignment and control of all aspects of the apparatus. These include 31 different servosystems to ensure optical, mechanical, and thermal stability.

\subsection{The Cs Experiment: Data and Results}

Approximately 350 hours of data on the PNC observables were acquired in five runs distributed over an 8-month period. Each of the five runs followed the same basic procedure. First, a set of auxiliary experiments was carried out to measure and set numerous quantities: (i) all three components of the average $\vec{E}$ and $\vec{B}$ fields and their $\hat{y}$ gradients in the interaction region; (ii) the magnitude and orientation of the birefringence of the PBC output-mirror coating; (iii) the polarization-dependent power modulation of the green laser light; and (iv) the populations of the $m_{F}$ levels of the atomic beam as it entered the interaction region. After these measurements were completed, the four laser frequencies were locked to the desired hyperfine transitions and data were acquired in blocks of about 1.5 hours each. During this time, five parity reversals - the electric field, laser polarization, and three ways of reversing the $m_{F}$ state being excited (reversing the polarization of the optical pumping light of laser 2, reversing the optical pumping magnetic field relative to the pumping light, and reversing the magnetic field in the $6 \mathrm{~S}-7 \mathrm{~S}$ excitation region) - were carried out at different rates. The electric field was reversed at $27 \mathrm{~Hz}$ and the others at various lesser rates. The relative phases of the various reversals were regularly shifted by one half cycle. Before and after each of the 1.5-hour blocks, the 
polarization of the 540-nm standing-wave field was measured and set. At regular intervals, the PBC output mirror was rotated by 90.0 degrees, and at irregular intervals, the frequencies of the four lasers were changed to measure PNC on the other $6 S$ - $7 S$ hyperfine line. At the end of each data run (20 to 30 1.5-hour blocks of data), the initial auxiliary experiments were repeated to check that the quantities described above had not changed significantly.

The typical size of the $6 S-7 S$ signal from the photodiode was $200 \mathrm{nA}$ on the 3-4 line and $85 \mathrm{nA}$ on the 4-3 line. These measurements corresponded to about $0.5 \%$ of the atomic beam undergoing a $6 S-7 S$ transition. The signal-to-noise ratio for measuring the $6 S-7 S$ transition rate was typically $55,000 / \sqrt{H z}$, and the parity violating modulation was typically about six parts in $10^{6}$. Because the technical noise was small and many photons were detected from each atom that had undergone a $6 S-7 S$ excitation, the noise was dominated by the shot-noise fluctuations in the number of atoms making the $6 S-7 S$ transition. Data was taken over a range of polarization ellipticities and electric fields.

The data analysis used to find the PNC modulation for each block of data was relatively simple. Appropriate combinations of fractional differences in the signal sizes were calculated for each of the 32 different field configurations to find the fraction of the rate that modulated with all five reversals. From the measured values of electric field and laser polarization this fractional modulation was then converted into $\operatorname{Im}\left(E 1_{P N C}\right) / \beta$. Various other small calibration corrections were required.

The major concern in this experiment was possible systematic errors arising from spurious signals that modulate under all five parity reversals, thus mimicking PNC. Roughly 20 times more data were taken in the investigation and elimination of such errors than in the actual PNC measurement. Several small errors associated with stray and misaligned fields were encountered, as in previous PNC measurements (24), and were treated as before. An exhaustive analysis was carried out of all possible combinations of static and oscillating electric and magnetic fields that could mimic a PNC signal. All of the stray (defined as nonreversing) and misaligned d.c. electric and magnetic fields and their gradients, and many of the laser field components, could be determined by looking at appropriate modulations in the $6 S-7 S$ rate under various conditions. Many of these quantities were extracted from the 31 different modulation combinations observed in real time while taking PNC data, and the remaining components were determined by the auxiliary experiments that were interspersed with the PNC runs. Many tests were also performed to ensure the necessary stability of the relevant fields.

Although the procedures were similar in concept to previous work, the Wood et al. (9) measurements were more difficult and time consuming due to the higher accuracy required. It proved necessary to consider not only the average fields, but also their gradients across the interaction region. Several small spurious signals were identified and removed. The absence of any systematic effects from the troublesome $A_{E}-A_{M 1}$ interference was confirmed by the independence of results 
for various laser polarizations sensitive to this interference. Other cross-checks included enhancing sources of error to confirm the predicted response and performing data analyses to verify that transition rate variations were consistent with fundamental shot-noise fluctuations.

The final results are

$$
\begin{array}{rll}
-\operatorname{Im}\left(\mathrm{E} 1_{\mathrm{PNC}}\right) / \beta & =1.6349 \pm 0.0080 \mathrm{mV} / \mathrm{cm} \quad 6 \mathrm{~S}_{\mathrm{F}=4} \leftrightarrow 7 \mathrm{~S}_{\mathrm{F}=3} \\
& =1.5576 \pm 0.0077 \mathrm{mV} / \mathrm{cm} \quad 6 \mathrm{~S}_{\mathrm{F}=3} \leftrightarrow 7 \mathrm{~S}_{\mathrm{F}=4}
\end{array}
$$

yielding for the nuclear-spin-dependent difference of interest for the anapole moment

$$
\operatorname{Im}\left(\mathrm{E} 1_{\mathrm{PNC}}^{\text {s.d. }}\right) / \beta=0.077 \pm 0.011 \mathrm{mV} / \mathrm{cm},
$$

a $7 \sigma$ effect. The statistical uncertainties for the two transitions, 0.0078 and 0.0073 $\mathrm{mV} / \mathrm{cm}$, respectively, dominate the error. The systematic uncertainties are based on statistical uncertainties in the determination of various calibration factors and systematic shifts, and therefore, it is appropriate to add them in quadrature. The final results are in good agreement with previous measurements in cesium but are much more precise. From the best available atomic calculation $(28,29)$ one then finds

$$
\kappa\left({ }^{133} \mathrm{Cs}\right)=0.112 \pm 0.016,
$$

a result we will find is dominated by the anapole moment.

\subsection{The Tl Experiment}

Experiments measuring parity violation in atomic thallium $\left(70.5 \%{ }^{205} \mathrm{Tl}, 29.5 \%\right.$ ${ }^{203} \mathrm{Tl}$ ) have not yet detected a nuclear spin dependent/anapole moment contribution, but they have achieved accuracies very near the level where anapole effects are expected. We will see that the resulting limits are interesting from the perspective of hadronic PNC. An interference of parity-allowed and PNC contributions to an atomic transition is observed, just as in cesium. However, in thallium the transition is an allowed magnetic dipole transition, $6 P_{1 / 2} \leftrightarrow 6 P_{3 / 2}$. The PNC interference is between the allowed M1 transition amplitude and the PNC E1 amplitude arising from weak interaction effects that mix $S$ states into the $P_{1 / 2}$ ground state. As Tl has an $I=1 / 2$ nuclear ground state, the $P_{1 / 2}$ state has $F=0$ and $F=1$ hyperfine sublevels which will be affected differently by nuclear-spin-dependent sources of PNC. Thus the search for spin-dependent PNC effects requires a comparison of the strength of PNC transitions out of the $F=0$ and $F=1$ states. Groups at the University of Washington (11) and Oxford (12) have made such comparisons.

The optical rotation of linearly polarized light that is produced by the interference of $E 1$ and $M 1$ transitions is measured in a large vapor cell of atomic thallium, as shown schematically in Fig. 5. A beam of linearly polarized laser light passes through the vapor and then through a nearly crossed linear polarizer followed by a sensitive detector. A Faraday modulator rotates the plane of polarization back and 


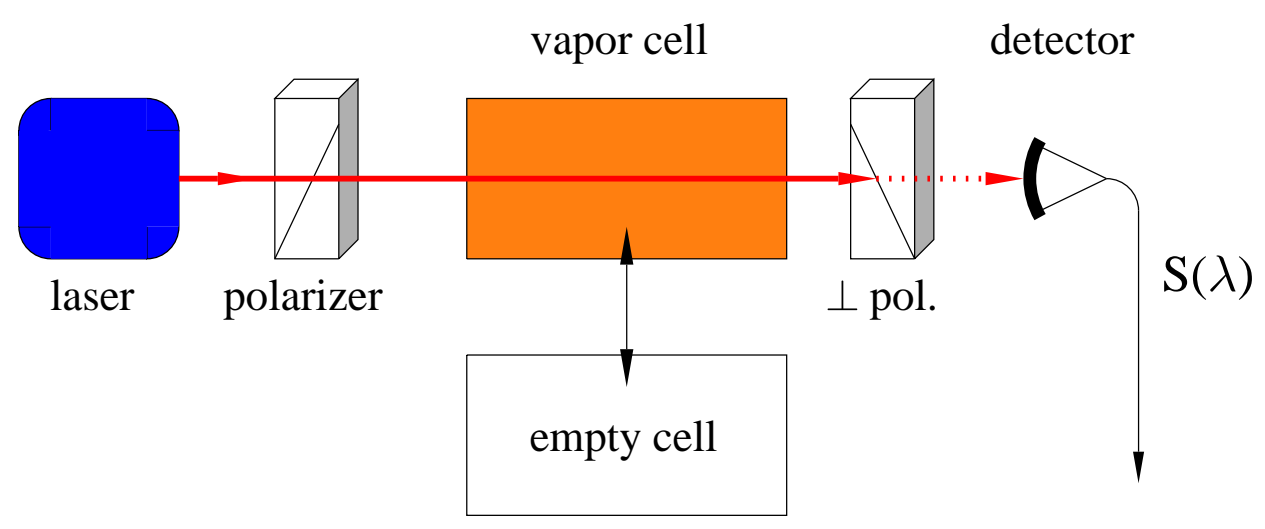

Figure 5: Schematic of the thallium vapor cell apparatus.

forth through the perfectly crossed position. This allows phase-sensitive detection that minimizes sensitivity to drifts in the signal baseline. The laser is scanned repeatedly across the transition and the detected light signal is then fit to the predicted lineshape. The PNC rotation reverses sign as the laser is tuned through line center. From the fit to the dispersion-like line shape the amplitude of the rotation can be determined. To distinguish the signal of interest from spurious frequencydependent rotations in optics, the vapor cell is interchanged with a "dummy" cell that has identical optics but no atomic vapor. An example of the data obtained in this fashion is shown in Fig. 6, taken from Ref (11).

It can be seen that the agreement between predicted and observed signals is excellent. As in the cesium experiment hundreds of hours of data were taken and considerable effort was made to eliminate possible systematic errors. The thallium oven was carefully constructed and shielded to avoid magnetic fields that would cause spurious Faraday rotation. Data were acquired over a wide range of pressures of thallium and a variety of tests of the calibration were carried out.

The final results of the experiments are expressed as the ratio of parity violating $E 1$ to magnetic dipole $M 1$ transition amplitudes, $R=\operatorname{Im}\left(\mathrm{E} 1_{\mathrm{PNC}} / \mathrm{M} 1\right)$. The nuclear-spin-independent PNC signals obtained are $R^{\text {s.i. }}=(-14.68 \pm 0.17) \times$ $10^{-8}$ (11) (normalized to ${ }^{205} \mathrm{Tl}$ ) and $(-15.68 \pm 0.45) \times 10^{-8}$ (12), corresponding to $1.2 \%$ and $2.9 \%$ accuracy. The nuclear-spin-dependent effects are consistent with zero,

$$
\begin{aligned}
R^{\text {s.d. }}(\mathrm{Tl}) & =(0.15 \pm 0.20) \times 10^{-8} \text { Seattle } \\
& =(-0.04 \pm 0.20) \times 10^{-8} \text { Oxford }
\end{aligned}
$$

The resulting constraints on $\kappa$ are

$$
\kappa(\mathrm{Tl})=0.29 \pm 0.40 \text { Seattle }
$$




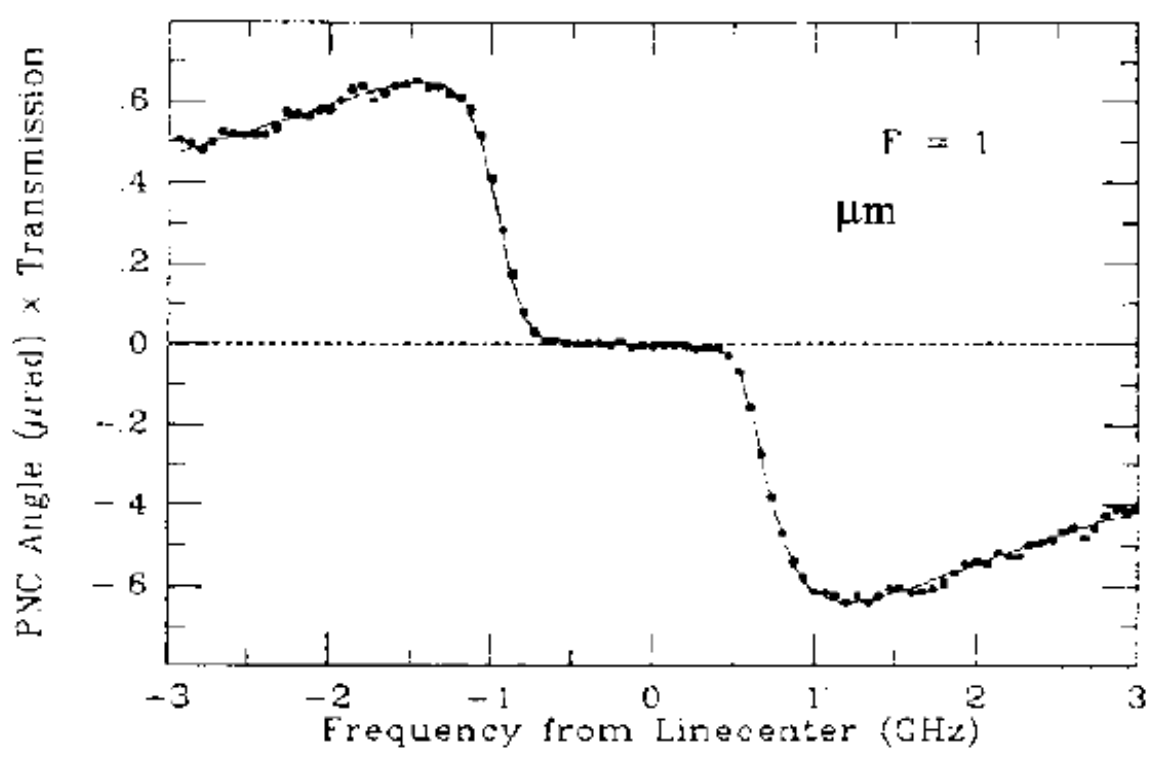

Figure 6: Combined PNC optical rotation for the thallium data cycles (a total of $\sim$ $230 \mathrm{~h}$ ) for $F=1$ (points) compared to the theoretical (dispersion $\times$ transmission) line shape (solid line).

$$
=-0.08 \pm 0.40 \text { Oxford }
$$

The ${ }^{133} \mathrm{Cs}$ and $\mathrm{Tl}$ spin-dependent measurements involve odd-proton nuclei, which leads to the prediction that they test a similar combination of isospin components of the weak hadronic PNC potential, as we will see below. Note that an anapole measurement for an odd-neutron nucleus would test a roughly orthogonal combination of isoscalar and isovector contributions to the hadronic PNC potential. Several atomic PNC efforts are underway that could produce such constraints (30,31).

\section{ANAPOLE MOMENTS AND HADRONIC PNC}

In this section we discuss the extraction of $\kappa_{\text {anapole }}$ from $\kappa$, the relation between $\kappa_{\text {anapole }}$ and the weak hadronic interaction, and the mechanisms by which a nucleus generates an anapole moment. 


\subsection{Extracting the Anapole Moment}

We have seen that atomic PNC measurements place the following constraints on the strength of nuclear-spin-dependent electron-nucleus interaction

$$
\begin{aligned}
\kappa\left({ }^{133} \mathrm{Cs}\right) & =0.112 \pm 0.016 \\
\kappa(\mathrm{Tl}) & =0.29 \pm 0.40 .
\end{aligned}
$$

where we have used the Seattle Tl result above because, due to its central value, it proves to be the more restrictive in the weak meson-nucleon parameter region favored by nuclear PNC experiments. The three principal contributions to $\kappa$

$$
\kappa=\kappa_{\text {anapole }}+\kappa_{Z^{0}}+\kappa_{Q_{W}}
$$

arise from the nuclear anapole moment, the vector(electron)-axial(nucleus) treelevel $Z^{0}$ exchange, and a term generated by the combined effects of the coherent $Z^{0}$ and magnetic hyperfine interactions between the electrons and the nucleus. In the theory discussions below we will treat the $\mathrm{Tl}$ constraint as one on the principal isotope, ${ }^{205} \mathrm{Tl}(70.5 \%)$. The other isotope, ${ }^{203} \mathrm{Tl}(29.5 \%)$, differs in structure only by a pair of neutrons, and thus should have very similar properties.

The tree-level vector(electron)-axial(nucleus) $Z^{0}$ interaction generates a contribution

$$
\kappa_{Z^{0}}=-\frac{g_{A}}{2}\left(1-4 \sin ^{2} \theta_{W}\right) \frac{<I\left\|\sum_{i=1}^{A} \sigma(i) \tau_{3}(i)\right\| I>}{<I|| \hat{I}|| I>},
$$

where $g_{A}=1.26$ is the axial-vector coupling and $\sin ^{2} \theta_{W}=0.223$. To get a rough feel for this contribution, we can evaluate the nuclear matrix element in the extreme single-particle limit. ${ }^{133} \mathrm{Cs}$ would then be described as a unpaired $1 g_{7 / 2}$ proton outside a closed core, while ${ }^{205} \mathrm{Tl}$ corresponds to an unpaired $3 s_{1 / 2}$ proton. In this limit

$$
\kappa_{Z^{0}}^{\text {single particle }}=-(-1)^{I-\ell-m_{t}} \frac{g_{A}}{2 \ell+1}\left(1-4 \sin ^{2} \theta_{W}\right)
$$

where $\ell$ is the single-particle orbital angular momentum and $m_{t}$ the $z$-component of isospin (with $m_{t}=1 / 2$ denoting a proton). Thus the single-particle estimates for ${ }^{133} \mathrm{Cs}$ and ${ }^{205} \mathrm{Tl}$ are 0.0151 and -0.136 , respectively. Nuclear models of various types have been employed to try to estimate the effects of strong correlations in quenching the Gamow-Teller matrix element in Eq. (24) from its single- particle value (19, 32, 33, 34). We will employ the results of a recent large-basis shell model (SM) study (32) here, which yielded

$$
\begin{aligned}
\kappa_{Z^{0}}^{S M}\left({ }^{133} \mathrm{Cs}\right) & =0.0140 \\
\kappa_{Z^{0}}^{S M}\left({ }^{205} \mathrm{Tl}\right) & =-0.127 .
\end{aligned}
$$

(We will describe these calculations in more detail later.) In addition, one-loop standard model electroweak radiative corrections, which we do not include in the 
numerical results below, modify the tree-level expression in Eq. (24) somewhat, reducing the isovector contribution and inducing a small isoscalar amplitude (35).

A second contribution to $\kappa$ is generated by the combined effects of the coherent vector $Z^{0}$ coupling to the nucleus, proportional to the weak charge $Q_{W}$, and the magnetic hyperfine interaction (36). From the measured nuclear weak charge and magnetic moment Bouchiat and Piketty find (37)

$$
\begin{aligned}
\kappa_{Q_{W}}\left({ }^{133} \mathrm{Cs}\right) & =0.0078 \\
\kappa_{Q_{W}}\left({ }^{205} \mathrm{Tl}\right) & =0.044 .
\end{aligned}
$$

Thus the experimental values for the anapole contributions to $\kappa$ are obtained by subtracting the results of Eqs. (26) and (27) from Eqs. (22), yielding

$$
\begin{aligned}
\kappa_{\text {anapole }}\left({ }^{133} \mathrm{Cs}\right) & =0.090 \pm 0.016 \\
\kappa_{\text {anapole }}\left({ }^{205} \mathrm{Tl}\right) & =0.376 \pm 0.400 .
\end{aligned}
$$

\subsection{The Hadronic Weak Interaction}

The various mechanisms operating within the nucleus to generate $\kappa_{\text {anapole }}$ arise from the hadronic weak interaction. As we noted in the introduction, the only practical strategy for studying the effects of $Z^{0}$ exchange between quarks is the investigation of the PNC $N N$ interaction. As anapole moments are now measureable in high precision atomic PNC experiments, they now become a part of that strategy.

The low-energy hadronic weak interaction can be described by a phenomenological current-current Lagrangian (14)

$$
L=\frac{G_{F}}{\sqrt{2}}\left(J_{W}^{\dagger} J_{W}+J_{Z}^{\dagger} J_{Z}\right)+\text { h.c. }
$$

where $J_{W}$ and $J_{Z}$ are the charged and neutral weak currents, respectively. If one considers only the light-quark $(u, d, s)$ contributions to these currents, then $J_{W}$ has two components

$$
J_{W}=\cos \theta_{C} J_{W}^{0}+\sin \theta_{C} J_{W}^{1}
$$

where $\sin \theta_{C} \sim 0.22$. The current $J_{W}^{0}$ drives the $u \rightarrow d$ transition and transforms as $\Delta I=1, \Delta S=0$, while $J_{W}^{1}$ drives the $u \rightarrow s$ transition and transforms as $\Delta I=1 / 2, \Delta S=-1$. (Here $I$ denotes isospin and $S$ strangeness.) The neutral current also has two components, $J_{Z}^{0}$ and $J_{Z}^{1}$, which transform as $\Delta I=0, \Delta S=0$ and $\Delta I=1, \Delta S=0$, respectively. The $\Delta S=0 N N$ interaction is then governed by the following piece of Eq. (29)

$$
\begin{aligned}
L^{\Delta S=0} & =\frac{G_{F}}{\sqrt{2}}\left[\cos ^{2} \theta_{C}\left(J_{W}^{0}\right)^{\dagger} J_{W}^{0}+\sin ^{2} \theta_{C}\left(J_{W}^{1}\right)^{\dagger} J_{W}^{1}\right. \\
& \left.+\left(J_{Z}^{0}\right)^{\dagger} J_{Z}^{0}+\left(J_{Z}^{1}\right)^{\dagger} J_{Z}^{1}+\left(J_{Z}^{0}\right)^{\dagger} J_{Z}^{1}+\left(J_{Z}^{1}\right)^{\dagger} J_{Z}^{0}\right]+ \text { h.c. }
\end{aligned}
$$


An important aspect of this equation is its isospin content. The symmetric product of two $J_{W}^{0}(\Delta I=1)$ currents transform as $\Delta I=0$ and 2 , while the symmetric product of two $J_{W}^{1}(\Delta I=1 / 2)$ currents transforms as $\Delta I=1$. Thus the $\Delta I=1$ component of the charged current weak $N N$ interaction is suppressed by $\tan ^{2} \theta_{C}$ compared to the $\Delta I=0,2$ components, while the neutral current $\Delta I=1$ contribution is unsuppressed. It follows that the neutral current should dominate this isospin channel.

The main goals of the field have been to isolate this neutral current and, more generally, to understand the mechanism by which the weak force is communicated over the relatively long distances characterizing low-energy $N N$ scattering or $N N$ interactions within the nucleus. While the standard model specifies the elementary couplings of the weak bosons to quarks, these vertices are dressed by the strong interaction to form couplings between physical particles, such as mesons to nucleons. We know, from the $\Delta I=1 / 2$ rule in strangeness-changing hadronic weak interactions (the strong enhancement of the ratio of $\Delta I=1 / 2$ to $3 / 2$ amplitudes), that strong interaction effects can substantially alter couplings from their underlying bare values.

The low-energy $N N$ weak interaction is conventionally described in a one-mesonexchange model, where one meson-nucleon vertex is weak and the other strong: this long-distance mechanism dominates at nuclear densities. Six weak couplings, $f_{\pi}$, $h_{\rho}^{0}, h_{\rho}^{1}, h_{\rho}^{2}, h_{\omega}^{0}$, and $h_{\omega}^{1}$, characterize the strengths of the isovector $\pi$, isoscalar/isovector/isotensor $\rho$, and isoscalar/isovector $\omega$ weak meson-nucleon couplings (13). This model is not as restrictive as it may first appear. First, CP invariance forbids any coupling between neutral $J=0$ mesons and on-shell nucleons, eliminating a number of candidate interactions (38). Second, the most general low-energy PNC interaction contains only five independent $S-P$ amplitudes. From this perspective, the description in terms of $\pi, \rho$, and $\omega$ exchange can be viewed as an effective theory, valid at momentum scales much below the inverse range of the vector mesons. At low momentum the detailed short-range behavior of the $S-P$ potentials is not resolvable: thus one could characterize the short-range weak interaction by five contact interactions corresponding to these independent $S-P$ amplitudes, supplemented by long-range $\pi$ exchange. The six meson-nucleon weak couplings allow one to mimic these six degrees of freedom.

Denoting the isoscalar strong meson-nucleon couplings by $g_{\pi N N}, g_{\rho}$, and $g_{\omega}$, the resulting $N N$ PNC potential is (14)

$$
\begin{aligned}
V^{P N C}(\vec{r}) & =\frac{i g_{\pi N N} f_{\pi}}{\sqrt{32} M}[\vec{\tau}(1) \times \vec{\tau}(2)]_{3}[\vec{\sigma}(1)+\vec{\sigma}(2)] \cdot \overrightarrow{\mathrm{u}}(\vec{r}) \\
& -\frac{1}{2 M}\left[g _ { \rho } \left(h_{\rho}^{0} \vec{\tau}(1) \cdot \vec{\tau}(2)+\frac{h_{\rho}^{1}}{2}\left[\tau_{3}(1)+\tau_{3}(2)\right]\right.\right. \\
& \left.+\frac{h_{\rho}^{2}}{2 \sqrt{6}}\left[3 \tau_{3}(1) \tau_{3}(2)-\vec{\tau}(1) \cdot \vec{\tau}(2)\right]\right)
\end{aligned}
$$


Table 2: Weak meson-nucleon coupling "best values" and "reasonable ranges" (in units of $10^{-6}$ ) from the standard-model calculations of Desplanques, Donoghue, and Holstein

\begin{tabular}{ccc}
\hline \hline Coupling & "Best Value" & "Reasonable Range" \\
\hline$f_{\pi}$ & 0.46 & $0.00 \rightarrow 1.14$ \\
$h_{\rho}^{0}$ & -1.14 & $1.14 \rightarrow-3.08$ \\
$h_{\rho}^{1}$ & -0.019 & $-0.038 \rightarrow 0.00$ \\
$h_{\rho}^{2}$ & -0.95 & $-0.76 \rightarrow-1.10$ \\
$h_{\omega}^{0}$ & -0.19 & $0.57 \rightarrow-1.03$ \\
$h_{\omega}^{1}$ & -0.11 & $-0.19 \rightarrow-0.08$ \\
\hline
\end{tabular}

$$
\begin{array}{ll}
\times & \left(\left(1+\mu_{\mathrm{v}}\right) i[\vec{\sigma}(1) \times \vec{\sigma}(2)] \cdot \overrightarrow{\mathrm{u}}_{\rho}(\vec{r})+[\vec{\sigma}(1)-\vec{\sigma}(2)] \cdot \overrightarrow{\mathrm{v}}_{\rho}(\vec{r})\right) \\
+ & g_{\omega}\left(h_{\omega}^{0}+\frac{h_{\omega}^{1}}{2}\left[\tau_{3}(1)+\tau_{3}(2)\right]\right) \\
\times & \left(\left(1+\mu_{\mathrm{s}}\right) i[\vec{\sigma}(1) \times \vec{\sigma}(2)] \cdot \overrightarrow{\mathrm{u}}_{\omega}(\vec{r})+[\vec{\sigma}(1)-\vec{\sigma}(2)] \cdot \overrightarrow{\mathrm{v}}_{\omega}(\vec{r})\right) \\
+ & \left.\frac{1}{2}\left[\tau_{3}(1)-\tau_{3}(2)\right][\vec{\sigma}(1)+\vec{\sigma}(2)] \cdot\left[g_{\omega} h_{\omega}^{1} \overrightarrow{\mathrm{v}}_{\omega}(\vec{r})-g_{\rho} h_{\rho}^{1} \overrightarrow{\mathrm{v}}_{\rho}(\vec{r})\right]\right]
\end{array}
$$

where $\vec{r}=\vec{r}_{1}-\vec{r}_{2}, \overrightarrow{\mathrm{u}}=\left[\vec{p}, e^{-m r} / 4 \pi r\right], \overrightarrow{\mathrm{v}}=\left\{\vec{p}, e^{-m r} / 4 \pi r\right\}$, and $\vec{p}=\vec{p}_{1}-\vec{p}_{2}$, with $\vec{r}_{1}$ and $\vec{p}_{1}$ the coordinate and momentum of nucleon 1 . This expression is usually evaluated assuming vector dominance, which fixes the strong scalar and vector magnetic moments, $\mu_{s}=-0.12$ and $\mu_{v}=3.70$.

"Best values" and broad "reasonable ranges" for the weak meson-nucleon couplings were defined some time ago by Donoghue, Desplanques, and Holstein (13) $(\mathrm{DDH})$, who deduced standard-model estimates for these vertices by using techniques like factorization, the quark model, and current algebra and sum rule methods. The broad "reasonable ranges" reflect the large degree of uncertainty implicit in such approximate tools, as well as the potential consequences of missing physics, such as strange-quark amplitudes (39). Nevertheless, the DDH results in Table 2 have provided experimentalists with benchmarks for PNC experiments.

In an ideal world one would determine the low-energy $N N S-P$ amplitudes, or equivalently the six weak meson-nucleon couplings, by a series of $N N$ scattering experiments. Such experiments require measurements of asymmetries $\sim 10^{-7}$, which is the natural scale for the ratio of weak and strong amplitudes, $4 \pi G_{F} m_{\pi}^{2} / g_{\pi N N}^{2}$. Only a single $N N$ measurement, the longitudinal analyzing power $A_{z}$ for $\vec{p}+p$, has been successful (40,41, 42). (Experiments have been done at 13.6, 45, and 221 MeV.) These results have been supplemented by a number of PNC measurements in nuclear systems, where accidental degeneracies between pairs of opposite-parity states can produce, in some cases, large enhancements in the PNC signal. The experiments include $A_{z}$ for $\vec{p}+\alpha$ at $46 \mathrm{MeV}$ (43), the circular polarization $P_{\gamma}$ of 
the $\gamma$-ray emitted from the $1081 \mathrm{keV}$ state in ${ }^{18} \mathrm{~F}(44)$, and $A_{\gamma}$ for the decay of the $110 \mathrm{keV}$ state in polarized ${ }^{19} \mathrm{~F}(45)$. It is widely agreed that these experiments can be interpretted relatively free of nuclear structure uncertainties: the systems are either few-body, where quasi-exact structure calculations can be done, or involve special nuclei where the PNC mixing matrix elements can be calibrated from axial-charge $\beta$ decay (46). An anaysis of these results, which have been in hand for some time, suggests that the isoscalar PNC $N N$ interaction - which is dominated by $\rho$ and $\omega$ exchange - is comparable to or slightly stronger than the DDH "best value," while the isovector interaction - dominated by $\pi$ exchange - is significantly weaker $(\lesssim 1 / 3)(14)$. As the isovector channel is expected to be enhanced by neutral currents, there has been great interest in confirming this result. The ${ }^{133} \mathrm{Cs}$ anapole result thus provides a possible crosscheck on this tentative conclusion that an isospin anomaly, superficially like the $\Delta I=1 / 2$ rule in flavor-changing decays, may exist in the $\Delta S=0$ weak $N N$ interaction.

\subsection{Weak Meson-Nucleon Couplings and the Anapole Moment}

We denoted in the introduction that an anapole moment - unlike the magnetic moment - of elementary fermions is not a gauge invariant quantity. The very nice discussion of this point offered by Musolf (21) ends with a very straightforward explanation: because PNC corrections to the electromagnetic current couple only to virtual photons, the amplitude for PNC photon emission is not a physical amplitude and thus need not be gauge independent. However the long-distance contributions to the anapole moment in the nucleus - the meson cloud contributions to the nucleon anapole moment and the many-body contributions due to the PNC $N N$ interaction and associated exchange currents - are both the dominant contribution to the nuclear anapole moment and separately gauge invariant (19). These contributions, associated with the weak meson-nucleon couplings, will now be discussed.

To evaluate the nuclear anapole moment in the context of some model of hadronic PNC one must take the ground-state expectation value of the operator in Eq. (9). If one works to first-order in the weak interaction, two types of terms contribute to the current matrix element. First are terms corresponding to the PNC weak contributions to $J_{1 \lambda}$. In the context of the weak meson-nucleon couplings, these include both one-body terms - mesonic loop corrections to the ordinary electromagnetic current involving one weak and one strong vertex, as illustrated in Fig. 7a-and exchange currents (Fig. 7b), where the weak and strong vertices attach to different nucleons. We stress that if the anapole operator of Eq. (9) is employed, the exchange currents are model dependent in the sense that all constraints imposed by current conservation are already explicitly enforced. The argument sometimes heard that minimal substitution in simpler, independent-particle treatments somehow accounts for the exchange currents is incorrect: once Eq. (9) is employed, the surviving exchange currents depend on aspects of the $N N$ interaction not con- 
strained by current conservation.
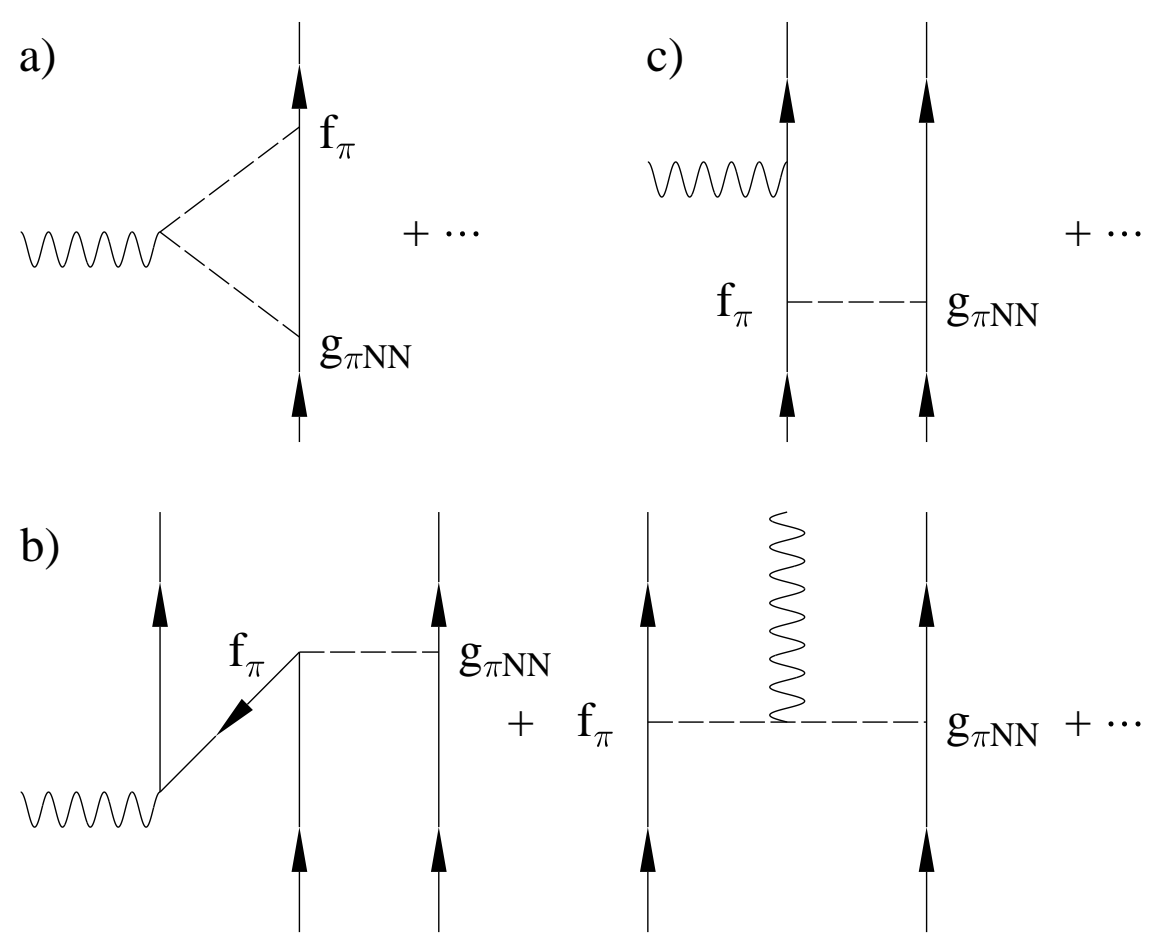

Figure 7: The one-body (a), exchange current (b), and nuclear polarization (c) contributions to the nuclear anapole moment.

The second class of contributions comes from evaluating Eq. (9) for the ordinary, parity-conserving $J_{1 \lambda}^{e m}$, which contributes through PNC admixtures in the nuclear wave function, as illustrated in Fig. 7c. The polarization term, which depends on the matrix elements of $V^{P N C}$ between the ground state and a complete set of excited opposite-parity states, can be enhanced if there exist opposite-parity states very near the ground state: the admixing is clearly inversely proportional to the energy difference (47). However, in the absence of such "accidental" degeneracies, one expects the mixing to be dominated by the giant resonances, the collective states at $\sim 15-20 \mathrm{MeV}$ in heavy nuclei that account for most of the $E 1$ and other first-forbidden nuclear response.

We discuss each of these contributions below, depending rather heavily on the recent work of Ref. (32). In Ref. (32) an effort was made to model the various contributions to the anapole moment using the formalism that has become standard in other studies of hadronic PNC: the DDH weak-meson couplings, the two-body 
$V^{P N C}$ based on those couplings, large-basis SM nuclear wave functions, and a standard correlation function to modified the short-distance behavior of the SM two-nucleon density. Thus this treatment will allow us, in Section 5, to compare anapole, $N N$, and nuclear constraints on hadronic PNC on an equal footing.

a) Nucleon anapole moment. The one-body PNC electromagnetic current derived from meson loop corrections (e.g., Fig. 7a) yields in the nonelativistic limit

$$
\hat{A}_{1 \lambda}^{\text {nucleonic }}=\sum_{i=1}^{A}\left[a_{s}(0)+a_{v}(0) \tau_{3}(i)\right] \sigma_{1 \lambda}(i) .
$$

That is, this term is the sum of the anapole moments of the individual nucleons. As the contribution of spin-paired core nucleons cancel, one expects this term to depend on the unpaired valence nucleon in the odd- $A$ nuclei of interest. Thus the one-body contribution should be roughly independent of $A$ though - like the closely related example of magnetic moments - its value will depend on the shells occupied by the valence nucleon. Some time ago the pion contribution to $a_{s}(0)$ and $a_{v}(0)$ was evaluated (19), yielding results proportional to $e f_{\pi} g_{\pi N N}$. The isoscalar coupling $a_{s}(0)$ proved to be about four times larger than $a_{v}(0)$. Recent extensions of this work have included the full set of one-loop contributions involving the DDH vector meson PNC couplings, using the framework of heavy baryon chiral perturbation theory and retaining contributions through $O\left(1 / \Lambda_{\chi}^{2}\right)$, where $\Lambda_{\chi}=4 \pi F_{\pi} \sim 1$ $\mathrm{GeV}$ is the scale of chiral symmetry breaking (35). The contributions due to $f_{\pi}$ are consistent with the earlier work - the new $a_{s}(0)$ is about 1.3 times larger, while $a_{v}(0)$ is zero in this order - so that the dominance of $a_{s}(0)$ in Ref. (19) was is explained as a consequence of heavy baryon chiral perturbation theory. The addition in Ref. (35) of the heavy meson contributions greatly enhances $a_{v}(0)$. An evaluation with DDH best value couplings yields $a_{v}(0) \sim 7 a_{s}(0)$. Thus the inclusion of heavy meson contributions substantially enhances the one-body anapole terms and alters the isospin character, generating opposite signs for the proton and neutron anapole moments.

This then determines $\hat{A}_{1 \lambda}^{\text {onebody }}$. The SM studies of Ref. (32), which we will describe in more detail below, give matrix elements $\left\langle I|| \sum_{i=1}^{A} \sigma(i)|| I\right\rangle=-2.37$ and 2.53 and $\left\langle I|| \sum_{i=1}^{A} \sigma(i) \tau_{3}(i)|| I\right\rangle=-2.30$ and 2.28 for ${ }^{133} \mathrm{Cs}$ and ${ }^{205} \mathrm{Tl}$, respectively. This leads to the nucleonic anapole contributions shown in Table 3 .

b) Exchange currents. Two-body PNC currents arise from meson exchange diagrams where the photon couples to the meson "in flight" (transition currents), or where the photon and either the PC or PNC meson-nucleon vertex creates and annihilates an $N \bar{N}$ pair (pair currents), as illustrated in Fig. 7b. The pionic exchange currents, which have the longest range, were evaluated in Ref. (19). The explicit form of the pion contribution to $A_{1 \lambda}^{e x}$ cur is given there. Rather than quote the result, which is a bit complicated, we instead give the one-body reduction of the pair current contribution to that operator, which illustrates the underlying physics much more clearly.

If one views a nucleus as a single-particle outside of a closed core, a two-body 
Table 3: Shell model estimates of the anapole matrix element $\left\langle I\left\|A_{1}\right\| I\right\rangle / e$, expressed as cofficients times the indicated weak couplings

\begin{tabular}{ccrrrrrr}
\hline \hline Nucleus & Source & $f_{\pi}$ & $h_{\rho}^{0}$ & $h_{\rho}^{1}$ & $h_{\rho}^{2}$ & $h_{\omega}^{0}$ & $h_{\omega}^{1}$ \\
\hline${ }^{133} \mathrm{Cs}$ & nucleonic & 0.59 & 0.87 & 0.90 & 0.36 & 0.28 & 0.29 \\
& ex. cur. & 8.58 & 0.02 & 0.11 & 0.06 & -0.57 & -0.57 \\
& polariz. & 51.57 & -16.67 & -4.88 & -0.06 & -9.79 & -4.59 \\
& total & 60.74 & -15.78 & -3.87 & 0.36 & -10.09 & -4.87 \\
$205 \mathrm{Tl}$ & nucleonic & -0.63 & -0.86 & -0.96 & -0.35 & -0.29 & -0.29 \\
& ex. cur. & -3.54 & -0.01 & -0.06 & -0.03 & 0.28 & 0.28 \\
& polariz. & -13.86 & 4.63 & 1.34 & 0.08 & 2.77 & 1.27 \\
& total & -18.03 & 3.76 & 0.33 & -0.30 & 2.76 & 1.26 \\
\hline
\end{tabular}

operator can be replaced by an equivalent one-body effective operator

$$
<\alpha\left|A_{1 \lambda}^{e f f}\right| \beta>=\sum_{\delta<F}<\alpha \delta\left|A_{1 \lambda}^{e x}{ }^{\text {cur }}\right| \beta \delta-\delta \beta>,
$$

where the sum is taken over the nuclear core. Completing this sum in Fermi gas model, assuming a spin-symmetric but isospin-asymmetric core, yields the pionic pair current effective operator

$$
\begin{aligned}
A_{1 \lambda}^{e f, N \bar{N}} & =2.74 a_{s}^{\pi}(0) \frac{M}{m_{\pi}^{2}} \sum_{i=1}^{A} \rho\left(r_{i}\right) r_{i}^{2}\left(\sigma_{1 \lambda}(i)+\sqrt{2 \pi}\left[Y_{2}\left(\hat{r}_{i}\right) \otimes \sigma(i)\right]_{1 \lambda}\right) \\
& \times\left(\frac{Z}{A} \omega_{Z}^{\pi}\left(1-\frac{2}{3} \tau_{3}(i)\right)+\frac{N}{A} \omega_{N}^{\pi}\left(1+\frac{2}{3} \tau_{3}(i)\right)\right),
\end{aligned}
$$

where $\rho\left(r_{i}\right)$ is the nuclear density operator and $\omega_{Z}^{\pi}\left(\omega_{N}^{\pi}\right)$ a proton (neutron) Fermigas response function that depends on $k_{i} / k_{F}$, the nucleon momentum as a fraction of the Fermi momentum. The $\omega$ s vary only gently, ranging from 0.33 to 0.19 as $k_{i} / k_{F}$ increases from 0 to 1 . Thus a suitable average value is $\sim 0.25$.

The overall strength of $A_{1 \lambda}^{e f f, N \bar{N}}$ is given in terms of the pionic contribution to the single-particle anapole moment

$$
a_{s}^{\pi}(0) \sim-1.6 \frac{e f_{\pi} g_{\pi N N}}{8 \sqrt{2} \pi^{2}}
$$

to allow an easy comparison with the one-body current. Using a nuclear density of $0.195 / \mathrm{fm}^{3}$, one finds that the pair isoscalar $\pi$ exchange current then scales as $\sim 0.9 A^{2 / 3} a_{s}^{\pi}(0)$. Unlike the nucleonic contribution, the exchange current contribution grows as $A^{2 / 3}$. Clearly it will increasingly dominate over the nucleonic contribution as $A$ increases. The isovector effective operator is smaller by a factor 
of $2(Z-N) / 3 A$, reflecting a cancellation between contributions from core protons and neutrons.

The calculations of Ref. (19) employed the full two-body form for the pionic currents, evaluating these from the SM two-body density matrices. A short-range correlation function was introduced to mimic the effects of hard-core correlations on the density matrix. The extension to include the $\rho$ and $\omega$ PNC couplings is a formidable task requiring evaluation of the $\rho$ and $\omega$ pair currents and the $\rho \rho \gamma$ and $\rho \pi \gamma$ currents. This calculation was only recently completed (32). The $\rho \rho \gamma$ and $\rho \pi \gamma$ transition currents and the component of the $\omega$ pair current where the photon and PNC $\omega$ couplings are on different nucleon legs were found to be negligible, well below $1 \%$ of the dominant $\pi$ currents; the remaining heavy-meson currents are more important but, as can be seen in Table 3, still are suppressed relative to the pionic currents. The tabulated results were obtained from the SM calculations described below.

c) Nuclear polarization contribution. The nuclear polarization contribution (Fig. 7c) to the anapole moment is given by

$$
\sum_{n} \frac{\left\langle I|| \hat{A}_{1}^{e m}|| n\right\rangle\left\langle n\left|H^{P N C}\right| I\right\rangle}{E_{g s}-E_{n}}+\text { h.c. }
$$

where $\hat{A}_{1}^{e m}$ is generated from inserting the ordinary electromagnetic current operator into Eq. (9), $|I\rangle$ is a ground state of good parity, $H^{P N C}$ is the PNC NN interaction, and the sum extends over a complete set of nuclear states $n$ of angular momentum $I$ and opposite parity. The extended Siegert's theorem again determines the form of $\hat{A}_{1}^{e m}(19)$.

Dmitriev, Khriplovich, and Telitsin (48) have provided a series of estimates of the polarization contribution in the context of single particle models, using a onebody effective $V^{P N C}$. The models include one of uniform density and ones based on the harmonic oscillator and Woods-Saxon potentials. The former yields an anapole operator that is explicitly proportional to the nuclear density - a quantity approximately constant in heavy nuclei because of nuclear saturation - and grows as $A^{2 / 3}$, like the exchange current contribution. Numerically, however, the polarization contribution is about a factor of five larger than the exchange currents: it is the dominant contribution to axial PNC in heavy nuclei.

For the present analysis we need a treatment of polarization contribution that begins with the DDH form of $V^{P N C}$. This presents two nuclear structure challenges. The first is the construction of a reasonable model for the ground state. The SM calculations of Refs. (19, 32) for ${ }^{133} \mathrm{Cs}$ were done in the canonical space between magic shells 50 and $82,1 g_{7 / 2}-2 d_{5 / 2}-1 h_{11 / 2}-3 s_{1 / 2}-2 d_{3 / 2}$. The protons were restricted to the first two of these shells and neutron holes to the last three, producing an m-scheme basis of about 200,000. ${ }^{205} \mathrm{Tl}$ is described as a proton hole in the orbits immediate below the $\mathrm{Z}=82$ closed shell $\left(3 s_{1 / 2}-2 d_{3 / 2}-2 d_{5 / 2}\right)$ coupled to two neutron holes in valence neutron space between magic numbers 126 and 82 $\left(3 p_{1 / 2}-2 f_{5 / 2}-3 p_{3 / 2}-1 i_{13 / 2}-2 f_{7 / 2}-1 h_{9 / 2}\right)$. 
The second challenge is the completion of the intermediate state sum. Direct or moments methods (19) are impractical because of the dimensions of the spaces. However, because no nonzero $E 1$ transitions exist among the valence orbitals, an alternative of completing the sum by closure, after replacing $1 / E_{n}$ by an average value $\langle 1 / E\rangle$, is quite attractive: the resulting product of the one-body operator $A_{1}^{e m}$ and two-body $V^{P N C}$ contracts to a two-body operator, so that only the twobody ground state density matrix is needed. The closure aproximation can be considered an identity if one knows the correct $\langle 1 / E\rangle$ : in practical terms, this means demonstrating that it can be related reliably to some known quantity, with the position of the $E 1$ giant resonance being the obvious candidate. A series of SM calculations has been completed for a series of light nuclei, where both the $1 / E-$ and non-energy-weighted sums could be done (32). It was found that there was a consistent relationship between the closure energy and the giant dipole position, though the three isospin contributions to $V^{P N C}$ must be treated separately. The average excitation energies found (as fractions of the dipole energy) are $0.604 \pm$ $0.056\left(h_{\rho}^{0}, h_{\omega}^{0}\right), 0.899 \pm 0.090\left(f_{\pi}\right)$, and $1.28 \pm 0.14\left(h_{\rho}^{2}\right)$. The larger $\langle 1 / E\rangle$ for $h_{\rho}^{0}$ and $h_{\omega}^{0}$ enhances these contributions to the anapole polarizability. The assumption that these relations also hold (47) in the heavier nuclei ${ }^{133} \mathrm{Cs}$ and ${ }^{205} \mathrm{Tl}$ then fixes the appropriate average excitation energies for these nuclei. From the SM two body density matrix, the contracted two-body effective operator that results from the closure approximation (this includes the effects of a short-range correlation function

on the two-nucleon matrix elements of $V^{P N C}$ ), and these excitation energies, one obtains the SM polarization results in Table 3 .

Note that there are situations - chance ground-state parity doublets or nuclear octupole deformation - that can lead to enhanced polarizabilities. While such enhancement is unexpected in ${ }^{133} \mathrm{Cs}$ as the nucleus is approximately spherical, enhancements have been discussed in connection with other nuclei of interest because of anapole or electric dipole moments (47).

\section{WEAK COUPLING CONSTRAINTS}

From Table 3 one has the matrix element of $A_{1 \lambda}$ as an expansion in terms of the DDH weak couplings. By Eq. (12), one has a corresponding expression for $\kappa_{\text {anapole }}$, which can be compared to the experimental constraints (Eq. (28)).

The constraints from ${ }^{133} \mathrm{Cs}$ and $\mathrm{Tl}$ supplement those available from experiments in $\vec{p}+p$ scattering and in nuclear systems. While a larger set of results exists, only some of these are generally regarded as being reliably interpretable (14). Rather precise measurements of the longitudinal analyzing power $A_{z}$ for $\vec{p}+p$ have been made at 13.6 and $45 \mathrm{MeV}$, and a preliminary result at $221 \mathrm{MeV}$ (where only $\rho$ exchange contributes) is now available. $A_{z}$ has also been measured for $\vec{p}+\alpha$ at 46 $\mathrm{MeV}$. There are also two important constraints from nuclei in which observables associated with nearly degenerate parity doublets have been measured. In each case the nuclear matrix element involved in the mixing has been determined from 
axial-charge $\beta$ decay (14, 46), so that little nuclear structure uncertainty remains. The observables are the circular polarization $P_{\gamma}$ of the $\gamma$-ray emitted in the decay of the $1081 \mathrm{keV}$ state in ${ }^{18} \mathrm{~F}$ and angular asymmetry $A_{\gamma}$ for the decay of the 110 $\mathrm{keV}$ state in polarized ${ }^{19} \mathrm{~F}$.

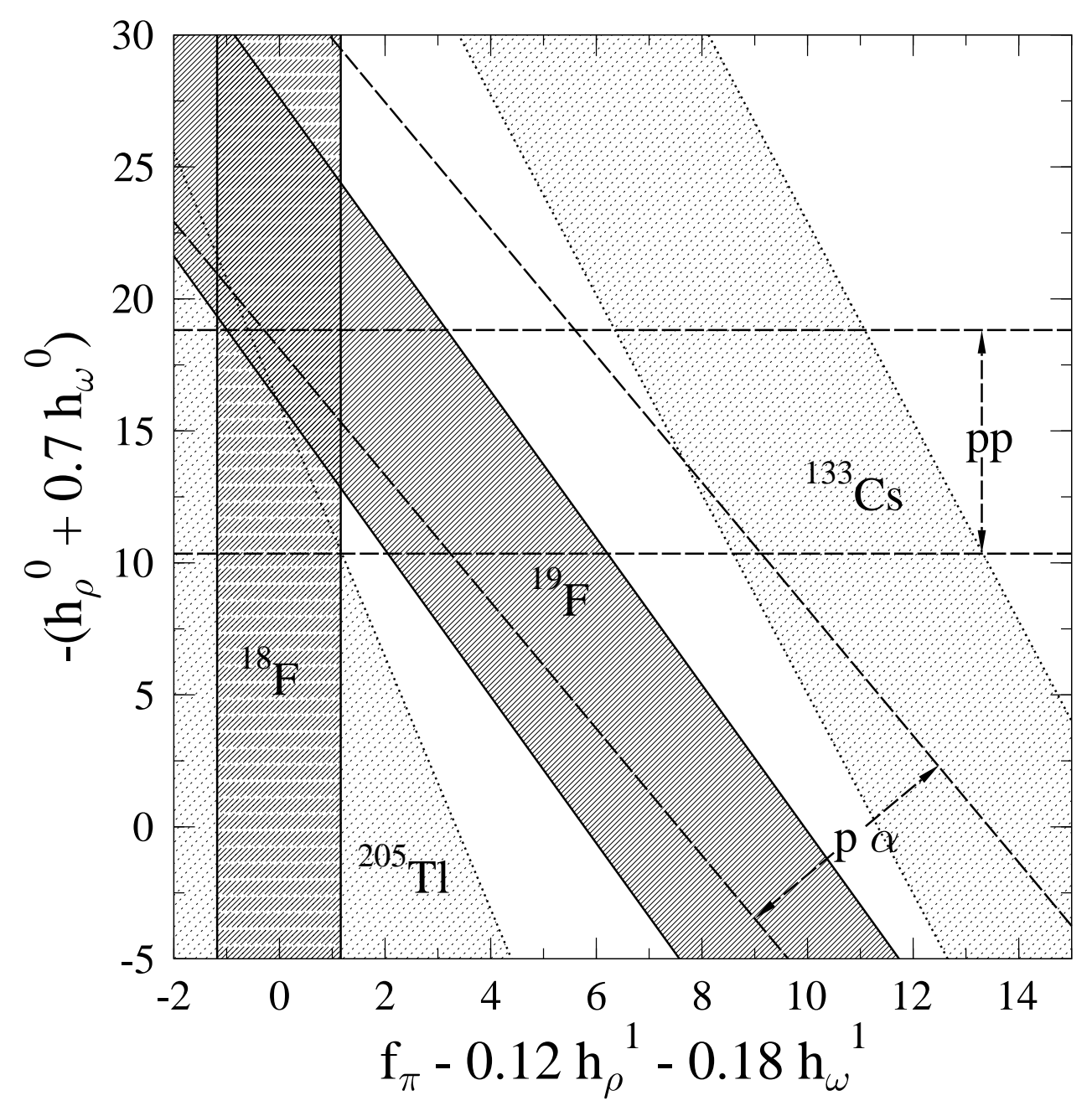

Figure 8: Constraints on the PNC meson couplings $\left(\times 10^{7}\right)$ that follow from the results in Table 1 . The error bands are one standard deviation. The illustrated region contains all of the DDH "reasonable ranges" for the indicated parameters.

A summary of PNC constraints is presented in Table 1 and Fig. 8. Although the PNC parameter space is six-dimensional, two coupling constant combinations, 
Table 4: PNC observables and corresponding theoretical predictions, decomposed into the designated weak-coupling combinations, with $\tilde{f}_{\pi}=f_{\pi}-0.12 h_{\rho}^{1}-0.18 h_{\omega}^{1}$ and $\tilde{h}^{0}=h_{\rho}^{0}+0.7 h_{\omega}^{0}$

\begin{tabular}{cccccccc}
\hline \hline Observable & Exp. $\left(\times 10^{7}\right)$ & $\tilde{f}_{\pi}$ & $\tilde{h}^{0}$ & $h_{\rho}^{1}$ & $h_{\rho}^{2}$ & $h_{\omega}^{0}$ & $h_{\omega}^{1}$ \\
\hline$A_{z}^{p p}(13.6)$ & $-0.93 \pm 0.21$ & & 0.043 & 0.043 & 0.017 & 0.009 & 0.039 \\
$A_{z}^{p p}(45)$ & $-1.57 \pm 0.23$ & & 0.079 & 0.079 & 0.032 & 0.018 & 0.073 \\
$A_{z}^{p p}(221)$ & prelim. & & -0.030 & -0.030 & -0.012 & 0.021 & \\
$A_{z}^{p \alpha}(46)$ & $-3.34 \pm 0.93$ & -0.340 & 0.140 & 0.006 & & -0.039 & -0.002 \\
$P_{\gamma}\left({ }^{18} \mathrm{~F}\right)$ & $1200 \pm 3860$ & 4385 & & 34 & & & -44 \\
$A_{\gamma}\left({ }^{19} \mathrm{~F}\right)$ & $-740 \pm 190$ & -94.2 & 34.1 & -1.1 & & -4.5 & -0.1 \\
$\left\langle\left\|A_{1}\right\|\right\rangle / e, \mathrm{Cs}$ & $800 \pm 140$ & 60.7 & -15.8 & 3.4 & 0.4 & 1.0 & 6.1 \\
$\left\langle\left\|A_{1}\right\|\right\rangle / e, \mathrm{Tl}$ & $370 \pm 390$ & -18.0 & 3.8 & -1.8 & -0.3 & 0.1 & -2.0 \\
\hline
\end{tabular}

$f_{\pi}-0.12 h_{\rho}^{1}-0.18 h_{\omega}^{1}$ and $h_{\rho}^{0}+0.7 h_{\omega}^{0}$, dominate the observables, as Table凹illustrates. The $1 \sigma$ error bands of Fig. 8 are generated from the experimental uncertainties, broadened somewhat by allowing uncorrelated variations in the parameters in the last four columns of Table 1 over the DDH broad "reasonable ranges." Note that only a fraction of the region allowed by the Seattle $\mathrm{Tl}$ constraint is shown: the total width of the $\mathrm{Tl}$ band is an order of magnitude broader than the width of the Cs allowed band, with most of the $\mathrm{Tl}$ allowed region lying outside the DDH "reasonable ranges" (i.e., in the region of negative $f_{\pi}$ and positive $h_{\rho}^{0}+0.7 h_{\omega}^{0}$ ). The corresponding Oxford $\mathrm{Tl}$ band, which is not illustrated, includes almost all of the parameter space in Fig. 8, as well as a substantial region (to the lower left of Fig. 8) outside the bounds of the figure.

The weak coupling ranges covered by Fig. 8 correspond roughly to the DDH broad "reasonable ranges." Thus the anapole constraints are not inconsistent with the most general theory constraints. However in detail, the pattern is disconcerting. Before the anapole results are included, the indicated solution is a small $f_{\pi}$ and an isoscalar coupling somewhat larger, but consistent with, the DDH best value, $-\left(h_{\rho}^{0}+0.7 h_{\omega}^{0}\right)_{b . v .}^{D D H} \sim 12.7$. The anapole results agree poorly with the indicated solution, as well as with each other. Although the Seattle Tl measurement is consistent with zero, it favors a positive anapole moment, while the theory prediction is decidedly negative, given existing PNC constraints. The Cs result tests a combination of PNC couplings quite similar to those measured in $A_{\gamma}\left({ }^{19} \mathrm{~F}\right)$ and in $A_{z}^{p \alpha}$, but favors larger values.

The results plotted in Fig. 8 for ${ }^{133} \mathrm{Cs}$ are consistent with those of Flambaum and Murray (28), who extract from the anapole moment an $f_{\pi}$ about twice the DDH best value, $f_{\pi b v .}^{D D H} \sim 4.6$, and point out that theory can accommodate this. (The DDH reasonable range is $0-11.4$, in units of $10^{-7}$.) However, this ignores $P_{\gamma}\left({ }^{18} \mathrm{~F}\right)$, a measurement that has been performed by five groups. The resulting 
constraint is almost devoid of theoretical uncertainty

$$
-0.6 \lesssim f_{\pi}-0.11 h_{\rho}^{1}-0.19 h_{\omega}^{1} \lesssim 1.2
$$

Allowing $h_{\rho}^{1}$ and $h_{\omega}^{1}$ to vary throughout their DDH reasonable ranges, one finds $-1.0 \lesssim f_{\pi} \lesssim 1.1$, clearly ruling out $f_{\pi} \sim 9$. Fig. 8 illustrates this, as well as the additional tension between $\mathrm{Cs}, p+\alpha$, and $A_{\gamma}\left({ }^{19} \mathrm{~F}\right)$.

\section{OUTLOOK}

The first conclusion one would draw from Fig. 8 is that additional experimental constraints would be helpful. In the case of nuclear experiments, the situation has been essentially static for the past 15 years, apart from the $\vec{p}+p A_{z}$ measurement at $221 \mathrm{MeV}$. However, in the next few years results are expected from experiments on the PNC spin rotation of polarized slow neutrons in liquid helium (49) and on $A_{\gamma}$ in $n+p \rightarrow d+\gamma$ (50). Thus we could soon have the information to test whether the small- $f_{\pi}$ solution favored by the $\vec{p}+p$ and nuclear experiments is correct: this is the one consistent solution satisfying the nuclear constraints.

The anapole situation is less satisfactory: even apart from the question of consistency with the nuclear constraints, there is some tension between the ${ }^{133} \mathrm{Cs}$ and Seattle Tl allowed regions, though Fig. 8 (which omits the bulk of the Tl allowed region) tends to exagerate this disagreement: if the $\mathrm{Tl}$ band were enlarged to $2 \sigma$, it would encompass all of the illustrated region, including the Cs band. While the Seattle Tl result favors a sign opposite theory, its broad error bar allows either sign. Taken together, the Seattle and Oxford results are not incompatible with any choice of coupling constants with the "reasonable ranges." An improved measurement in $\mathrm{Tl}$ would clearly be helpful, as would any new anapole measurement involving an odd-neutron nucleus, which would produce a band in Fig. 8 roughly perpendicular to that for Cs. There is an atomic PNC effort underway using Dy, which has two abundant odd-neutron isotopes, ${ }^{161}$ Dy and ${ }^{163}$ Dy (30). Another possibility may come from a new atomic PNC technique using a single trapped $\mathrm{Ba}^{+}$ion: the much larger coherence times and field intensities possible with this method compensates for the sensitivity loss stemming from the use of a single atom. (The statistical accuracy of traditional methods goes as $\sqrt{N}$, where $N$ is the number of atoms.) Ba has two stable odd-neutron isotopes, ${ }^{135} \mathrm{Ba}$ and ${ }^{137} \mathrm{Ba}$. As with Cs these attempts will have to reach the sub- $1 \%$ level of precision in order to permit an extraction of the differential effects due to nuclear spin dependence. Alternatively other methods, such as proposals to measure the anapole moment directly through $E 1 / M 1$ interference in hyperfine transitions (51), could be developed.

The underlying issues are not limited to atomic PNC measurements: our understanding of $\mathrm{V}(e)-\mathrm{A}(N)$ interactions affects the interpretation of electron-nucleus PNC scattering experiments like SAMPLE (52), where a similar discrepancy be- 
tween theory and experiment exists, one that could be associated with an incomplete treatment of PNC effects in the nuclear target.

Figure 8 shows that the jump in precision achieved in the ${ }^{133} \mathrm{Cs}$ experiment has now provided a constraint on hadronic PNC that is comparable in accuracy to the best of the nuclear constraints. This is the reason the lack of overlap in the resulting bands is a concern. The nuclear physics required to analyze the ${ }^{133} \mathrm{Cs}$ result is nontrivial (53), and could yet prove to be the source of the discrepancies apparent in Fig. 8. The SM calculations described in Section 4 depend on a relation between the closure energy and the giant dipole energy that has been tested only in light nuclei. It is possible that the systematics for neutron-rich nuclei might be different. It is also generally appreciated that spin-dependent operators tend to be quenched as model spaces are enlarged to encompass more realistic correlations. Indeed, phenomenological single-particle treatments of anapole moments have previously envoked phenomenological quenching factors (37). Our SM results are quenched relative to single-particle estimates, and would likely be further weakened if those calculations could be enlarged. Likewise core polarization results from the RPA study of Dmitriev and Telitsin find substantial quenching, relative to single-particle estimates (33). All of these results argue that more realistic treatments of correlations will tend to reduce matrix element values, thus requiring still larger values of the weak coupling constants to fit the experimental results. Despite this possibility, it is clear that the degree of effort required to complete the ${ }^{133} \mathrm{Cs}$ experiment obligates theorists to invest similar effort in improving their calculations. It is an unfortunate situation that the results in Table 3 come from model calculations: it is thus difficult to come up with any strategy for quantifying possible errors other than the comparisons that can be made when still more ambitious calculations are eventually performed.

This work was support in part by the US Department of Energy and by the National Science Foundation. WH thanks the Miller and Guggenheim Foundations for support they provided.

\section{Literature Cited}

1. Lee TD and Yang CN. Phys. Rev. 104:254 (1956)

2. Wu CS, Ambler E, Hayward RW, Hoppes DD, Hudson RP, et al. et al. Phys. Rev. 105:1413 (1957); Garwin R, Lederman LM, and Weinrich M. Phys. Rev. 105:1415 (1957); Friedman JI and Telegdi VL. Phys. Rev. 105:1681 (1957)

3. Zeldovich YaB. Sov. Phys. JETP 6:1184 (1958) and citations therein

4. Bouchiat M-A and Bouchiat C. Rep. Prog.Phys. 60:1351 (1997); Sandars PGH. Phys. Scripta 36:904 (1987)

5. Erler J and Langacker P. Phys. Rev. Lett. 84:212 (1990)

6. Casalbuoni R, DeCurtis S, Dominici D, Gatto R, and Riemann S. hep-ph/0001215

7. Ramsey-Musolf MJ. Phys. Rev. C 60:015501 (1999)

8. Flambaum VV and Khriplovich IB. Sov. Phys. JETP 52:835 (1980); Flambaum VV, Khriplovich IB, and Sushkov OP. Phys. Lett. B 146:367 (1984)

9. Wood CS, Bennett SC, Cho D, Masterson BP, Roberts JL, et al. Science 275:1759 (1997)

10. Noecker MC, Masterson BP, and Wieman CE. Phys. Rev. Lett. 61:310 (1988) 
11. Vetter P, Meekhof DM, Majumder PK, Lamoreaux SK, and Fortson EN. Phys. Rev. Lett. 74:2658 (1995)

12. Edwards NH, Phipp SJ, Baird PEG, and Nakayama S. Phys. Rev. Lett. 74:2654 (1995)

13. Desplanques B, Donoghue JF, and Holstein BR. Ann. Phys. (NY) 124:449 (1980)

14. Adelberger EG and Haxton WC. Annu. Rev. Nucl. Part. Sci. 35:501 (1985); Haeberli W and Holstein BR. Symmetries and Fundamental Interactions in Nuclei, ed. W. C. Haxton and E. M. Henley. World Scientific: Singapore (1996)

15. DeForest Jr T and Walecka JD. Ann. Phys. (NY) 15:1 (1966).

16. Kayser B. Phys. Rev. D 26:1662 (1982) and 30:1023 (1984); Nieves JF. Phys. Rev. D 26:3152 (1982); Dubovik VM and Kuznetzov VE. Int. J. Mod. Phys. A 13:5257 (1998)

17. Siegert AJF. Phys. Rev. 52:787 (1937)

18. Friar JL and Fallieros S. Phys. Rev. C 29:1654 (1984); Friar JL and Haxton WC. Phys. Rev. C 31:2027 (1985)

19. Haxton WC, Henley EM, and Musolf MJ. Phys. Rev. Lett. 63:949 (1989); Haxton WC. Science 275:1753 (1997)

20. Khriplovich IB. Parity Nonconservation in Atomic Phenomena. Gordon and Breach: Philadelphia (1991)

21. Musolf MJ. Electroweak Corrections to Low-Energy Parity-Violating Neutral Current Interactions, Ph.D. thesis, Princeton (1989)

22. Henley EM, Huffman A, and Yu D. Phys. Rev. D 7:943 (1973).

23. Bouchiat M-A and Bouchiat C. Phys. Lett. 48B:111 (1974); Barkov LM and Zolotorev M. JETP Lett. 27:357 (1978); Conti R, Bucksbaum P, Chu S, Commins E, and Hunter L. Phys. Rev. Lett. $42: 343$ (1979)

24. Gilbert SL, Noecker MC, Watts RN, and Wieman CE. Phys. Rev. Lett. 55:2680 (1985); Gilbert SL and Wieman CE. Phys. Rev. A 34:792 (1986)

25. Masterson BP, Tanner C, Patrick H, and Wieman CE. Phys. Rev. A 47:2139 (1993)

26. Wieman CE, Gilbert S, Noecker Ch, Masterson P, Tanner C, et al. Frontiers in Laser Spectroscopy, ed. T. Hansch and M. Inguscio. North-Holland: Amsterdam (1994); Drever RWP, Hall JL, Kowalski FV, Hough J, Ford GM, et al. Appl. Phys. B 31:97 (1983)

27. Wieman CE and Holberg L. Rev. Sci. Instrum. 62:1 (1991)

28. Flambaum VV and Murray DW. Phys. Rev. C 56:1641 (1997)

29. Dzuba VA, Flambaum VV, Silvestrov PG, and Sushkov OP. J. Phys. B 20:3297 (1987); Kraftmakher AYa. Phys. Lett. A 132:167 (1988)

30. Budker D. Physics Beyond the Standard Model, ed. P. Herczeg, C. M. Hoffman, and H. V. Klapdor-Kleingrothaus. World Scientific: Singapore (1998)

31. Fortson EN. Phys. Rev. Lett. 70:2383 (1993)

32. Haxton WC, Liu CP, and Ramsey-Musolf MJ. nucl-th/0101018

33. Dmitriev VF and Telitsin VB. Nucl. Phys. A 674:168 (2000)

34. Auerbach N and Brown BA. nucl-th/9903032

35. Musolf MJ and Holstein BR. Phys.Lett. B 242:461 (1990) and Phys. Rev. D 43:2956 (1991); Zhu S-L, Puglia SJ, Holstein BR, and Ramsey-Musolf MJ. Phys. Rev. D 62:033008 (2000); Maekawa CM, Veiga JS, van Kolck U. Phys. Lett. B 488:167 (2000)

36. Flambaum VV and Khriplovich IB. Sov. Phys. JETP 62:872 (1985); Kozlov MG. Phys. Lett. A 130:426 (1988)

37. Bouchiat C and Piketty CA. Z. Phys. C 49:91 (1991) and Phys. Lett. B 269:195 (1991)

38. Barton G. Nuovo Cimento 19:512 (1961)

39. Dai J, Savage MJ, Liu J, and Springer R. Phys. Lett. B 271:403 (1991)

40. Balzer R, Henneck R, Jacquemart Ch, Lang J, Nessi-Tedaldi F, et al. Phys. Rev. C 30:1409 (1984) and Phys. Rev. Lett. 44:699 (1980)

41. Potter JM, Bowman JD, Wang CF, McKibben JL, Mischke RE, et al. Phys. Rev. Lett. 33:1307 (1974); Nagle DE, Bowman JD, Hoffman C, McKibben JL, Mischke R, et al. 3rd International 
Symposium on High Energy Physics with Polarized Beams and Polarized Targets. AIP Conf. Proc. 51 (1978)

42. Ramsay WD, Birchall J, Bland J, Hamian AA, Lee L, et al. Phys. Canada 55:79 (1999)

43. Lang J, Maier Th, Muller R, Nessi-Tedaldi F, Roser Th, et al. Phys. Rev. Lett. 54:170 (1985)

44. Barnes CA, Lowry MM, Davidson JM, Marris RE, Morinigo FB, et al. Phys. Rev. Lett. 40:840 (1978); Bizetti PG, Fazzini TF, Maurenzig PR, Perego A, Poggi G, et al. Lett. Nuovo Cimento 29:167 (1982); Ahrens G, Harfst W, Kass JR, Mason EV, Schober H, et al. Nucl. Phys. A 390:496 (1982); Page SA, Evans HC, Ewan GT, Kwan S-P, Leslie JR, et al. Phys. Rev. C 35:1119 (1987) and Phys. Rev. Lett. 55:791 (1985); Bini M, Fazzini TF, Poggi G, and Taccetti N. Phys. Rev. Lett. 55:795 (1985)

45. Adelberger EG, Hindi MM, Hoyle CD, Swanson HE, vonLintig RD, et al. Phys. Rev. C 27:2833 (1987); Elsener K, Gruebler W, Konig V, Schmelzbach PA, Ulbricht J, et al. Nucl. Phys. A 461:579 (1987) and Phys. Rev. Lett. 52:1476 (1984)

46. Haxton WC. Phys. Rev. Lett. 46:698 (1981)

47. Haxton WC and Henley EM. Phys. Rev. Lett. 51:1937 (1983); Flambaum VV. Phys, Rev. A 60:R2611 (1999); Engle J, Friar JL, and Hayes AC. Phys. Rev. C 61:035502 (2000); Tsvetkov A, Kvasil J, and Nazmitdinov RG. nucl-th/0009085

48. Dmitriev VF, Khriplovich IB, and Telitsin VB. Nucl. Phys. A 577:691 (1994); Wilburn WS and Bowman JD. Phys. Rev. C 57:3425 (1998)

49. Heckel B. private communication

50. Snow WM, Bazhenov A, Blessinger CS, Bowman JD, Chupp TE, et al. Nucl. Instrum. Meth. A 440:729 (2000)

51. Gorshkov VG, Ezhov VF, Kozlov MG, and Mikhailov AI. Sov. J. Nucl. Phys. $48: 867$ (1988); Bouchiat M-A and Bouchiat C. physics/0101098

52. Hasty R, Hawthorne-Allen AM, Averett T, Barkhuff D, Beck DH, et al. Science 290:2117 (2000)

53. An example where anapole calculations can be done in a model-independent way is the deuteron effective field theory result: Savage MJ and Springer RP. Nucl.Phys. A 686:413 (2001); Savage MJ. nucl-th/0012043 